\title{
Effect of Magnesium Reduction Process on Oxygen Content of Pickling Niobium Powder
}

\section{Jingfeng Wang ( $\nabla$ wangjingfeng@zzrvtc.edu.cn )}

Zhengzhou Railway Vocational \& Technical College

\section{Fang Liu}

Zhengzhou University

\section{Yue Zhang}

Zhengzhou University

\section{Qinkui Li}

Zhengzhou University

\section{Research Article}

Keywords: pickling niobium powder, oxygen content, oxygen reduction process, magnesium reduction process

Posted Date: May 20th, 2021

DOI: https://doi.org/10.21203/rs.3.rs-526437/v1

License: (c) (1) This work is licensed under a Creative Commons Attribution 4.0 International License. Read Full License

Version of Record: A version of this preprint was published at Scientific Reports on July 22nd, 2021. See the published version at https://doi.org/10.1038/s41598-021-94578-7. 


\title{
Effect of magnesium reduction process on oxygen content of pickling niobium powder
}

\author{
Wang Jingfeng ${ }^{1}$, Liu Fang ${ }^{2}$, Zhang Yue ${ }^{2}$, Li Qinkui ${ }^{2}$, \\ 1. Zhengzhou Railway Vocational and Technical College, Zhengzhou, Henan, CN, 450000 \\ 2. Zhengzhou University, Zhengzhou, Henan, CN, 450000 \\ Corresponding author: wangjingfeng@zzrvtc.edu.cn
}

\begin{abstract}
Aiming at the problem of high oxygen content in industrial niobium powder, the oxygen reduction process of high oxygen niobium powder magnesium was studied. Based on the thermodynamic analysis of magnesium thermal reduction of niobium powder, the effects of reduction temperature, magnesium addition, reduction time and reduction atmosphere on oxygen content of pickling niobium powder were studied. The results show that: with the increase of magnesium addition, the oxygen content of pickling niobium powder gradually decreases to a certain value and then remains unchanged. In a certain temperature range (953k-1203k), with the increase of reduction temperature, the oxygen content of pickling niobium powder first decreases and then increases, and the best oxygen content is $356 \mathrm{ppm}$ at $1133 \mathrm{k}$; with the extension of reduction time (2-6h), the oxygen content of pickling niobium powder first decreases and then remains unchanged. Finally, the oxygen content of pickled niobium powder is reduced to about $356 \mathrm{ppm}$ at $400 \% \mathrm{Mg}$ addition, $1133 \mathrm{~K}$ reduction temperature and $4 \mathrm{~h}$ reduction time.
\end{abstract}

Keywords : pickling niobium powder, oxygen content, oxygen reduction process, magnesium reduction process

\section{Introduction}

Niobium and niobium alloys are widely used in electronic industry, aerospace industry, iron and steel industry, automobile industry, low temperature superconductor and medical diagnostic equipment due to their good physical, mechanical and electrical properties, such as good corrosion resistance and ductility, low density, high melting point, high specific strength, good conductivity and thermal conductivity, and small 
thermal neutron capture cross section. Niobium powder is an important raw material for the preparation of niobium and niobium alloy. At present, industrial niobium powder is mainly obtained by crushing niobium strip and hydrogenating. Although the particle size of niobium powder is relatively small, which is conducive to the compactness of powder metallurgy products, niobium powder with small particle size has high affinity with oxygen and is easy to adsorb oxygen, resulting in high oxygen content of niobium powder [1-5]. Too high oxygen content in niobium powder will seriously affect the physical, mechanical and electrical properties of niobium, thus restricting the application of niobium and niobium alloys [6-10].

Therefore, to solve the problem of high oxygen content in industrial niobium powder, it is urgent to find a process to reduce the oxygen content in niobium powder. The preparation of low oxygen and fine niobium powder by magnesium reduction of $\mathrm{Nb}_{2} \mathrm{O}_{5}$ has become a hot spot. Magnesium thermal reduction has the advantages of simple process, low energy consumption and easy separation of reaction products. However, there are few studies on magnesium reduction to reduce the oxygen content of niobium powder [11-15]. After oxygen reduction, the oxygen content of niobium powder is still on the high side, and the complete degree of oxygen binding with magnesium in the process of magnesium reduction and the degree of secondary oxidation on the surface of niobium powder in the process of cooling will affect the oxygen content of niobium [15-22].

In this work, the industrial niobium powder with $4100 \mathrm{ppm}$ oxygen content and 9.8 um Fisher particle size was studied. Firstly, the thermodynamics and kinetics of magnesium thermal reduction of niobium powder were analyzed to select the appropriate reduction temperature range. On this basis, the effects of magnesium addition, reduction time, reduction atmosphere and reduction temperature on oxygen content of pickling niobium powder were studied. Finally, the influence of two-step reduction method on oxygen content of pickling niobium powder was discussed.

\section{Experimental}

\subsection{Materials}


Hydrochloric acid 、 nitric acid 、hydrogen peroxide、 hydrofluoric acid and Anhydrous ethanol are analytically pure, purchased from Luoyang Haohua Chemical Reagent Co., Ltd (China). The purity of magnesium particles is $99.95 \%$, the particle size is $1-10 \mathrm{~mm}$, purchased from Aladdin Reagent Co., Ltd (USA). The niobium powder used in the experiment is provided by Ningxia tantalum industry, with oxygen content of $4100 \mathrm{ppm}$, particle size of $9.8 \mathrm{um}$ and specific surface area of $0.2 \mathrm{~m} 2 / \mathrm{g}$. The specific chemical composition is shown in Table 1.

Table 1 Chemical composition of niobium powder

\begin{tabular}{ccccccccc}
\hline component & $\mathrm{Nb}$ & $\mathrm{O}$ & $\mathrm{C}$ & $\mathrm{S}$ & $\mathrm{H}$ & $\mathrm{Fe}$ & $\mathrm{Si}$ & $\mathrm{Ti}$ \\
\hline content $/ \%$ & 99.48 & 0.41 & 0.0203 & 0.005 & 0.002 & 0.013 & 0.057 & 0.011 \\
\hline
\end{tabular}

\subsection{Sample preparation}

The $\mathrm{Nb}_{2} \mathrm{O}_{5}$ reaction between magnesium powder and niobium surface $\left(5 \mathrm{Mg}+\mathrm{Nb}_{2} \mathrm{O}_{5}=2 \mathrm{Nb}+5 \mathrm{MgO}\right)$ produces niobium and $\mathrm{MgO}[22]$. Theoretically, $1.5 \mathrm{~g}$

$\mathrm{Mg}$ is needed for $1 \mathrm{~g} \mathrm{O}$. Because magnesium is easy to volatilize due to high saturated vapor pressure during sintering, and oxygen is inevitably introduced in reduction atmosphere and operation process, the amount of magnesium should be excessive. According to the experimental basis in the early stage of the experiment, the amount of magnesium added in this experiment was 200\%, 300\%, 400\%, 500\% and $600 \%$ respectively. The meaning of excess is 2 times, 3 times, 4 times, 5 times and 6 times of the theoretical amount of magnesium needed for magnesium thermal reduction reaction. The specific experimental method is as follows: $15 \mathrm{~g}$ niobium powder and corresponding amount of magnesium are evenly mixed and put into the crucible, and then reduced in the hydrogenation and dehydrogenation furnace. After natural cooling to room temperature, the furnace is opened for material collection, and acid washing, drying and oxygen content analysis are carried out.

\subsection{Characterization}

The contents of oxygen, carbon and other impurities in niobium powder were 
determined by oxygen, nitrogen and hydrogen content analyzer (ONH-3000, Gangyannake Testing Technology Co., Ltd) and high frequency infrared carbon sulfur analyzer (CS-3000, Gangyannake Testing Technology Co., Ltd). The metal impurities $\mathrm{Fe}$, Ti and non-metal impurities Si in niobium powder were determined by inductively coupled plasma atomic analyzer (ICP-AES, ThermoFisher Scientific). XRD (6100, Shimadzu) was used to analyze the intermediate products in the reaction process and detect whether there is any biological phase change. The experimental conditions are $\mathrm{Cu}-\mathrm{K} \alpha$ ray, scanning speed is $2^{\circ} / \mathrm{min}$, scanning angle is $30 \sim 90^{\circ}$, working voltage is $40 \mathrm{kV}$, working current is $30 \mathrm{~mA}$, scanning mode is continuous scanning. Laser particle size analyzer (Mastersizer 3000, Malvern) and average particle size analyzer (WLLP208a, Dandong Fisher Instrument Co., Ltd.) were used to determine the particle size of niobium powder. SEM and XPS were used to analyze the effect of oxygen behavior on the morphology of niobium powder before and after oxygen reduction and the existing state of oxygen on the surface of original niobium powder. The morphology of niobium powder before and after oxygen reduction was characterized by field emission scanning electron microscope (Quant 250 FEG, USA Fei) and X-ray photoelectron spectroscopy (AXIS Supra, Shimadzu) The existence of oxygen on the surface of high oxygen niobium powder was analyzed.

\section{Results and discussion}

\subsection{Magnesium addition}

Different amount of magnesium can affect the sufficient degree of oxide reduction on the surface of niobium powder [23], and then affect the oxygen 
content of pickling niobium powder. Moreover, different Mg Addition will also affect the loose degree of reduction products, which will affect the subsequent pickling effect. Excessive mg will cause niobium powder to be wrapped into a block by molten mg during the cooling process. The reduction products with too serious sintering are not conducive to acid pickling in the later stage. Too loose niobium powder may be that magnesium has volatilized before the oxide on $\mathrm{Nb}$ surface is fully reduced, which leads to high oxygen content in pickling niobium powder. Therefore, we need to optimize the amount of magnesium.

The effects of $200 \%, 300 \%, 400 \%, 500 \%$ and $600 \%$ of theoretical magnesium addition on the oxygen content of acid washed niobium powder were studied under the conditions of argon reduction atmosphere, graphite crucible and $1133 \mathrm{~K}$ reduction for $4 \mathrm{~h}$. After acid washing, the reduced product was vacuum dried at $333 \mathrm{~K}$ for $6 \mathrm{~h}$, and the oxygen content was measured by $\mathrm{ONH}$ analyzer. The specific value of oxygen content is shown in Table 2 , and the change trend is shown in Figure 1. The macro morphology of the reduction products obtained under the conditions of original niobium powder and different magnesium addition is shown in Figure 2.

Table 2 Oxygen content of pickling niobium powder reduced at $1133 \mathrm{~K}$ for $4 \mathrm{~h}$ with different magnesium addition

\begin{tabular}{cccccc}
\hline Magnesium addition $/ \%$ & Original & 200 & 300 & 400 & 500 \\
\hline oxygen content $/ \mathrm{ppm}$ & 4100 & 481 & 412 & 356 & 349 \\
\hline
\end{tabular}




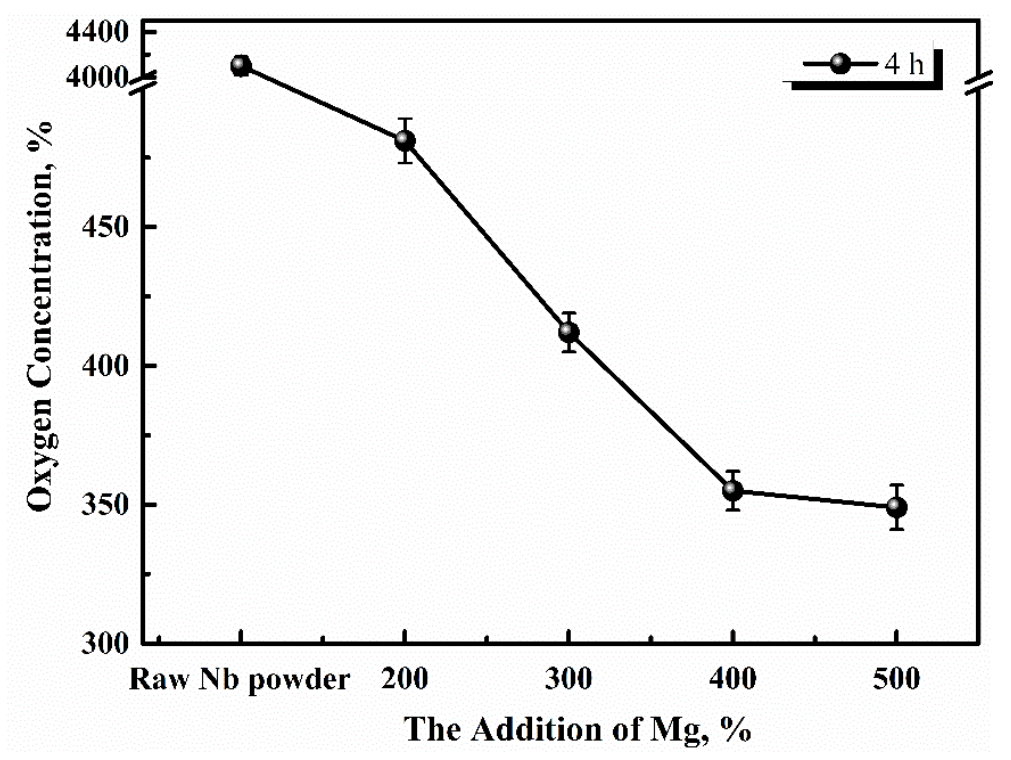

Figure 1 Effect of magnesium addition on oxygen content of pickling niobium powder at $1133 \mathrm{~K}$ for $4 \mathrm{~h}$
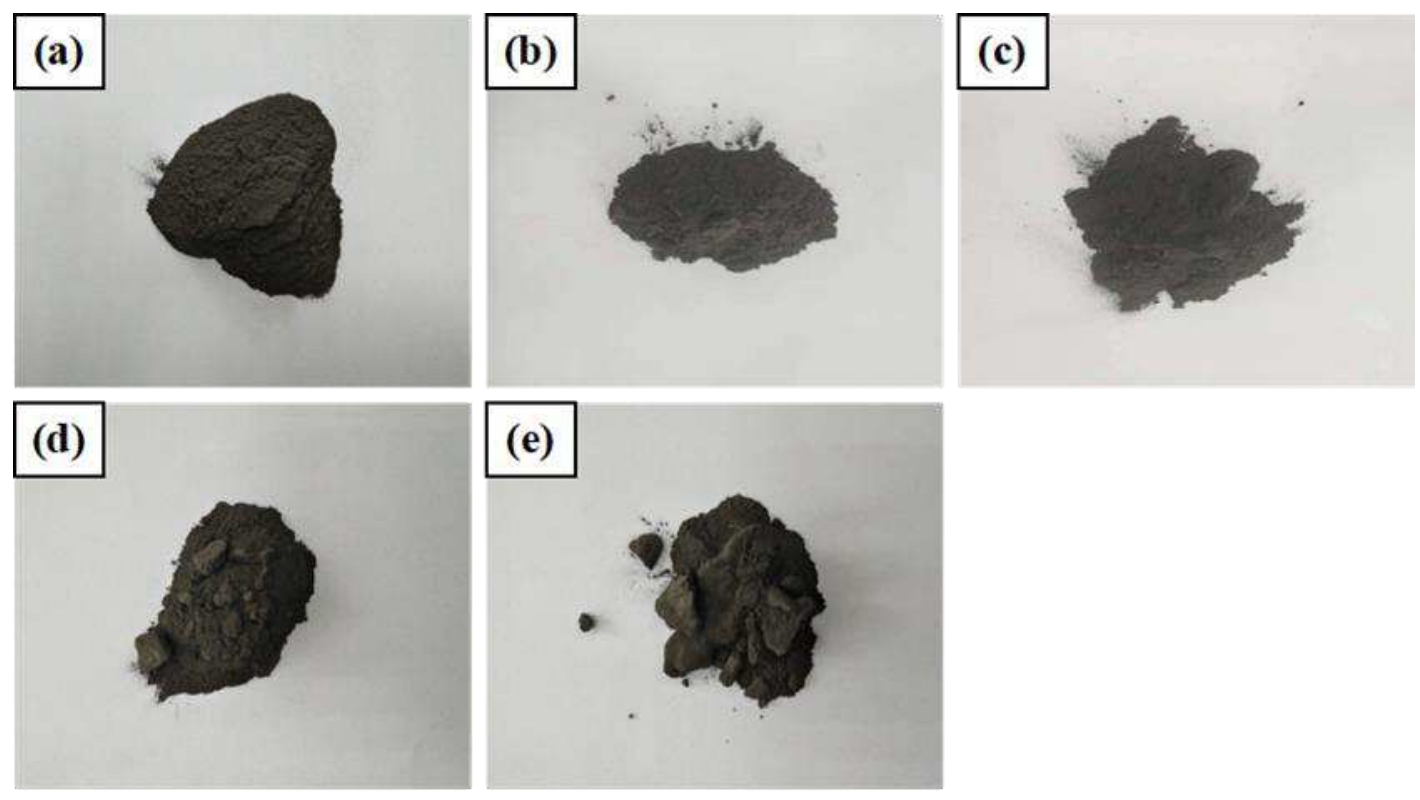

Figure 2 Different Mg Addition on morphology of reduced niobium powder

(a) original niobium powder, (b) $200 \%$ theoretical amount, (c) $300 \%$ theoretical amount, (d) 400\% theoretical amount, (e) 500\% theoretical amount According to Table 2, Figure 1 and Figure 2. As can be seen from Figure 2 (b), when the original high oxygen niobium powder is reduced at $200 \%$ 
magnesium content and $1133 \mathrm{~K}$ for $4 \mathrm{~h}$, the reduction product is loose without any caking phenomenon. There is a little residual magnesium chips on the surface of niobium powder, and the oxygen content of niobium powder obtained after pickling is $481 \mathrm{ppm}$. When the addition of magnesium is increased to $300 \%$, it can be seen from Figure2 (c) that some of the reduced products are slightly agglomerated, but the cohesion between the powders is very small, and the residual magnesium chips increase. At this time, the oxygen content of acid washed niobium powder is about 412ppm. With the increase of magnesium content to $400 \%$, it can be seen from Figure2 (d) that the reduction product is obviously formed and the hardness of the powder increases, but the powder can be broken with a little force, which will not cause difficulties to the subsequent pickling process. At this time, the oxygen content of the pickling niobium powder is about $356 \mathrm{ppm}$. When the addition of magnesium is increased to $500 \%$, it can be seen from Figure2 (e) that a large number of reduction products agglomerate. At this time, the oxygen content of the pickling niobium powder is $349 \mathrm{ppm}$, but the hardness of the reduction products is high, which brings difficulties to the subsequent pickling. It can be concluded that the oxygen content of pickling niobium powder is related to the loose degree and caking of reduced niobium powder. Under the condition of $1133 \mathrm{~K}$ reduction for $4 \mathrm{~h}$, the oxygen content of pickled niobium powder decreases to the same level with the increase of magnesium content from $200 \%$ to $500 \%$. When the magnesium content is $400 \%$, the loose degree of reduced niobium powder is the best, and the 
oxygen content of pickling niobium powder is the lowest, about $356 \mathrm{ppm}$. It can also be concluded from the loose state of reduction products corresponding to different magnesium additions that the agglomeration phenomenon at $1133 \mathrm{~K}$ for $4 \mathrm{~h}$ is not due to the self-sintering of niobium powder (without magnesium addition), but due to the solidification agglomeration during the cooling process caused by excessive magnesium.

\subsection{Effect of reduction temperature and time on oxygen content of pickling} niobium powder

The effects of reduction temperature and time on oxygen content of acid washed niobium powder were studied under the conditions of argon reduction atmosphere, graphite crucible, optimum pickling process and drying process. $953 \mathrm{~K}, 1053 \mathrm{~K}, 1093 \mathrm{~K}$ and $1133 \mathrm{~K}$ were selected to study, and then the effects of different reduction time and magnesium addition amount on oxygen content of pickling niobium powder were studied at a specific reduction temperature. The experimental results are shown in Table 3, 4, 5, 6 and Figures 3, 4, 5,6

Table 3 Oxygen content of pickling niobium powder after reduction at $953 \mathrm{~K}$ for $8 \mathrm{~h}$ with different magnesium addition

\begin{tabular}{cccccc}
\hline Magnesium addition $/ \%$ & 50 & 100 & 150 & 200 & 300 \\
\hline oxygen content /ppm & 3600 & 2995 & 1600 & 890 & 874 \\
\hline
\end{tabular}




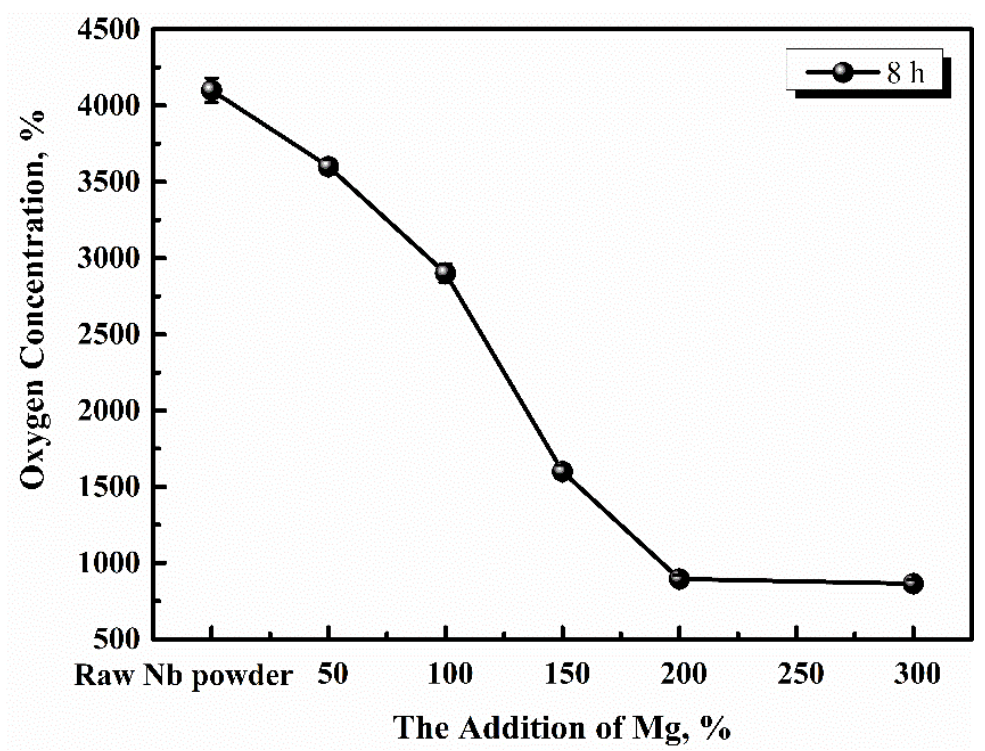

Figure 3 Oxygen content of pickling niobium powder at $953 \mathrm{~K}$ for $8 \mathrm{~h}$ with different magnesium addition

It can be seen from Table 3 and Figure 3 that when the reduction temperature is $953 \mathrm{~K}$ and the amount of magnesium is $50 \%$, the oxygen content of pickling niobium powder is $3600 \mathrm{ppm}$; when the amount of magnesium is increased to $200 \%$, the oxygen content of pickling niobium powder is reduced to $890 \mathrm{ppm}$; when the amount of magnesium is increased to $300 \%$, the oxygen content of pickling niobium powder is basically unchanged, about 874 ppm, indicating that $200 \%$ magnesium is enough. The results show that large particles of residual magnesium can be seen on the surface of $\mathrm{Nb}$ powder after reduction, which indicates that the magnesium thermal reduction reaction is not carried out thoroughly at this time. It can be seen that this is due to the low reduction temperature and slow reaction speed.

Table 4 Oxygen content (PPM) of acid washed niobium powder with different magnesium addition and reduction time at $1053 \mathrm{~K}$

\begin{tabular}{ccccc}
\hline Magnesium addition $/ \%$ & 200 & 300 & 400 & 500 \\
\hline reduction time $/ \mathrm{h}$ & & & & \\
\hline 2 & 588 & 536 & 541 & \\
3 & 571 & 529 & 538 & \\
4 & 560 & 520 & 530 & 524 \\
\hline
\end{tabular}




\begin{tabular}{llll}
\hline 6 & 525 & 527 & 528 \\
8 & 528 & 532 & 536 \\
\hline
\end{tabular}

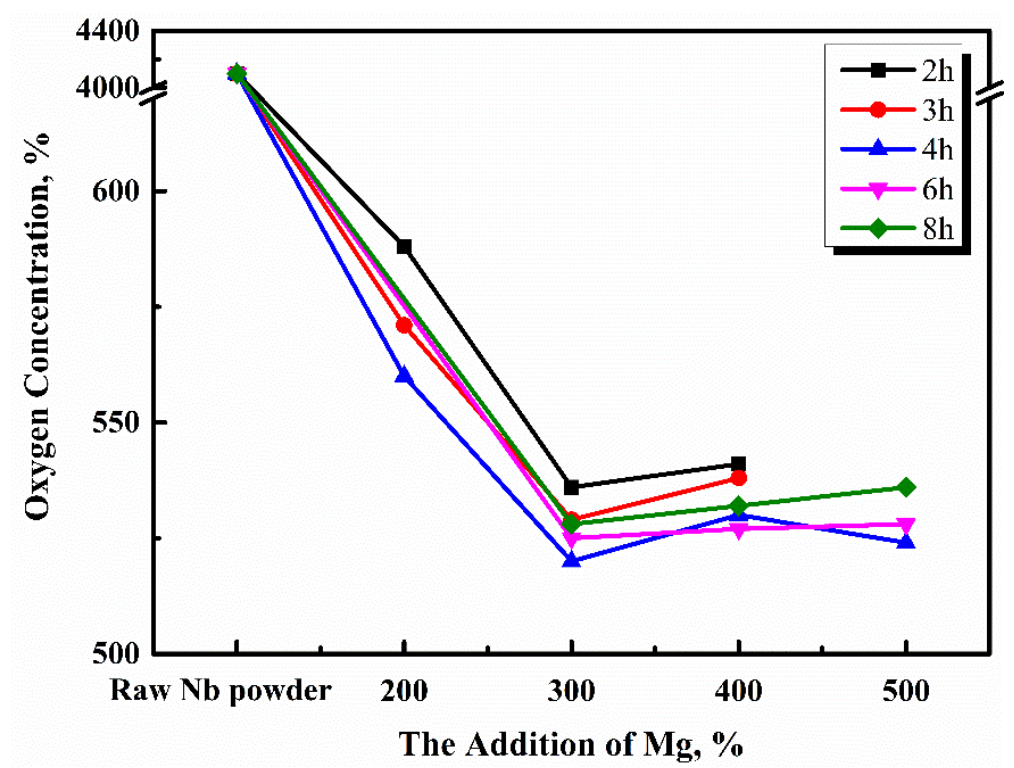

Figure4 Effect of magnesium addition and reduction time on oxygen content of pickling niobium powder at $1053 \mathrm{~K}$

It can be seen from Table 4 and Figure 4 that the oxygen content of pickled niobium powder is about $530 \mathrm{ppm}$ when the magnesium addition is $300 \%$ at $1053 \mathrm{~K}$ for $2 \mathrm{~h}, 3 \mathrm{~h}, 4 \mathrm{~h}, 6 \mathrm{~h}$ and $8 \mathrm{~h}$ reduction; with the extension of reduction time, the oxygen content of pickled niobium powder has no obvious change. When the reduction temperature increases from $953 \mathrm{~K}$ to $1053 \mathrm{~K}$, the oxygen content of acid washed niobium powder decreases about $350 \mathrm{ppm}$. It can be seen that with the increase of reduction temperature, the reaction rate of magnesium reducing $\mathrm{Nb}_{2} \mathrm{O}_{5}$ is accelerated, and the magnesium thermal reduction reaction is relatively sufficient. When the reduction time is prolonged, the oxygen content of acid washed niobium powder decreases less, which indicates that the reduction temperature at $1053 \mathrm{~K}$ is still low and the reaction speed is slow. The results show that the oxygen content of pickling niobium powder with $200 \%$ magnesium addition is significantly higher than that with $300 \%$ magnesium addition, but the oxygen content of pickling niobium powder with $400 \%$ and $500 \%$ 
magnesium addition has little change compared with that with 300\%. It shows that $300 \%$ excess magnesium is needed to meet the magnesium consumption in the reduction process at $1053 \mathrm{~K}$, which is higher than that at $953 \mathrm{~K}$. This is because the volatilization loss of magnesium increases with the increase of reduction temperature. At the same time, the addition of magnesium can not fully guarantee the oxygen content at $1053 \mathrm{~K}$ reduction.

Table 5 Oxygen content of pickling niobium powder at $1093 \mathrm{~K}$ with different magnesium addition and reduction time (PPM)

\begin{tabular}{ccccc}
\hline Magnesium addition $/ \%$ & 300 & 400 & 500 \\
\hline 2 & 200 & & 481 & 479 \\
3 & 560 & 490 & 460 & 463 \\
4 & 524 & 461 & 421 & 425 \\
5 & 508 & 433 & 406 & 408 \\
6 & 481 & 409 & 403 & 404 \\
\hline
\end{tabular}

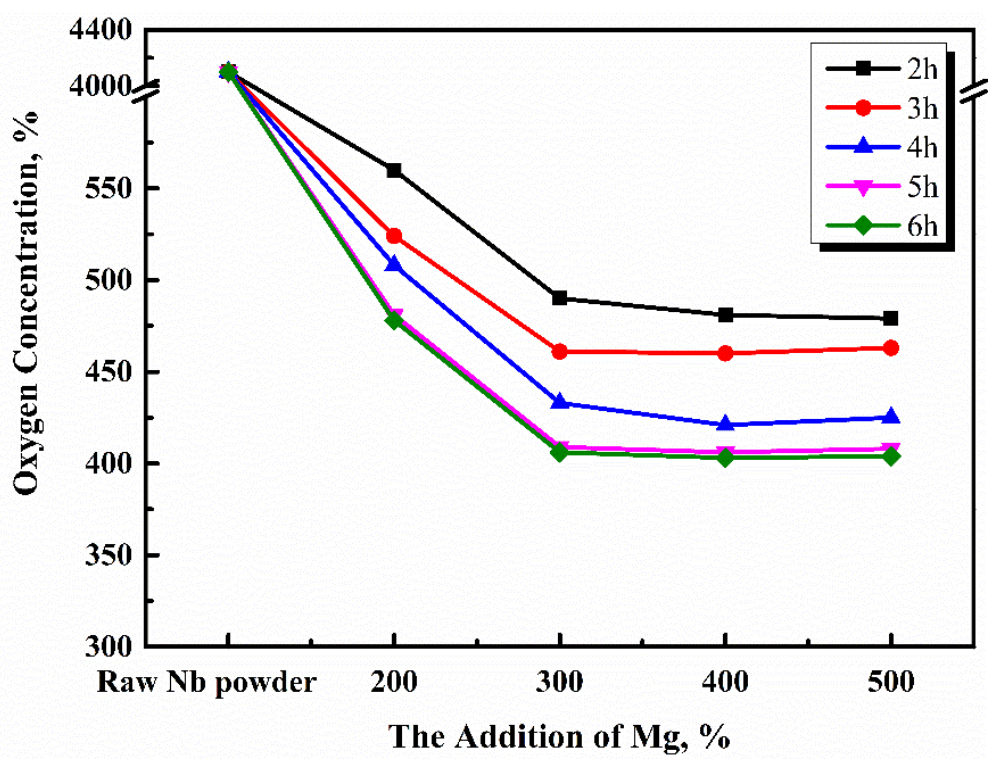

Figure 5 Effect of magnesium addition and reduction time on oxygen content of pickling niobium powder at $1093 \mathrm{~K}$

It can be seen from Table 5 and Figure 5 that when magnesium reduction temperature is $1093 \mathrm{~K}$, magnesium addition is $400 \%$ and reduction time is $2 \mathrm{~h}$, the oxygen content of pickling niobium powder is $481 \mathrm{ppm}$; when reduction time is $5 \mathrm{~h}$, the oxygen content of pickling niobium powder is $406 \mathrm{ppm}$, which may 
because the magnesium reduction reaction becomes more and more abundant with the extension of reduction time. When the reduction time is extended to 6 $\mathrm{h}$, the oxygen content of the acid washed niobium powder is about $403 \mathrm{ppm}$. Therefore, when the reduction time increases from $2 \mathrm{~h}$ to $6 \mathrm{~h}$, the oxygen content of acid washed niobium powder first decreases and then remains unchanged, and the lowest oxygen content of acid washed niobium powder is $406 \mathrm{ppm}$ after $5 \mathrm{~h}$ reduction. When the reduction temperature increases from $1053 \mathrm{~K}$ to $1093 \mathrm{~K}$, the oxygen content of pickled niobium powder decreases from $530 \mathrm{ppm}$ to 406 ppm, which is also due to the higher reduction temperature, the faster reduction rate of $\mathrm{Nb}_{2} \mathrm{O}_{5}$ by magnesium and the more sufficient reduction reaction. The oxygen content of pickling niobium powder is obviously lower than that of $200 \%$ magnesium addition when magnesium addition is over $300 \%$. When magnesium addition is increased to $400 \%$, the oxygen content of pickling niobium powder is basically unchanged. This shows that $300 \%$ magnesium addition is enough for magnesium consumption at $1093 \mathrm{~K}$ reduction temperature. At $1093 \mathrm{~K}$, the optimal magnesium reduction process parameters are $400 \%$ magnesium addition and $5 \mathrm{~h}$ pickling time. At this time, the oxygen content of pickling niobium powder is 406 ppm.

Table 6 Oxygen content of pickling niobium powder at $1133 \mathrm{~K}$ with different magnesium addition and reduction time (PPM)

\begin{tabular}{cccccc}
\hline Magnesium addition $/ \%$ & & & & \\
reduction time $/ \mathrm{h}$ & 200 & 300 & 400 & 500 & 600 \\
\hline 2 & 530 & 460 & 445 & 450 & \\
3 & 505 & 462 & 420 & 418 & \\
4 & 481 & 412 & 356 & 349 & \\
5 & & & 360 & 337 & 340 \\
6 & & & 357 & 356 & 353 \\
\hline
\end{tabular}




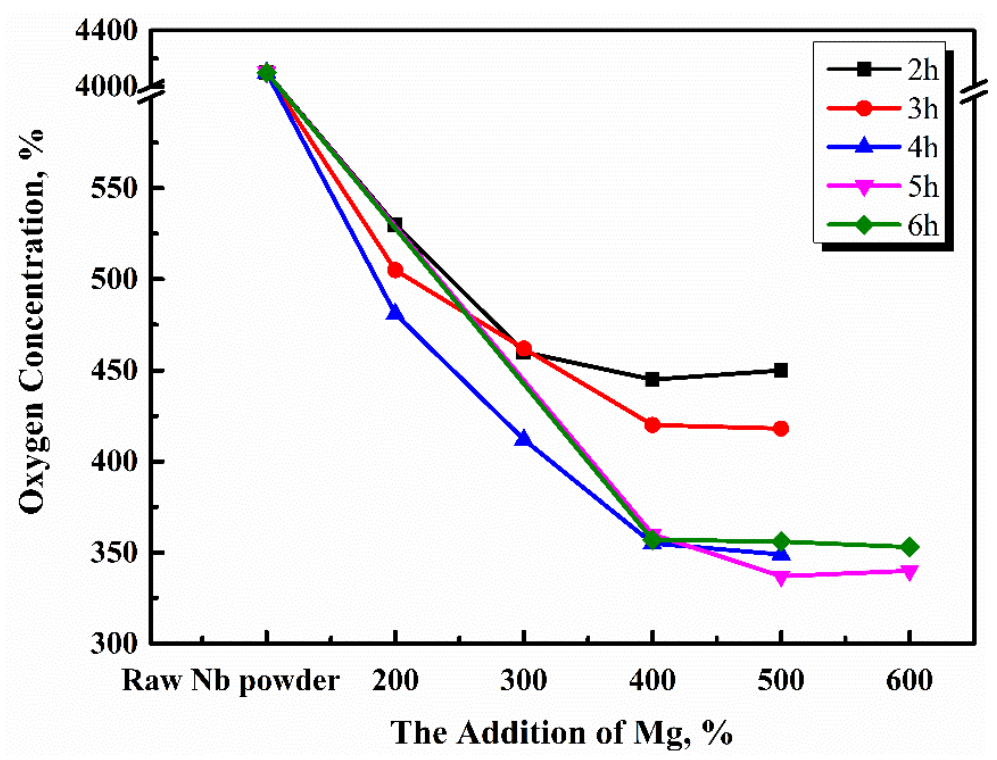

Figure 6 Effect of magnesium addition and reduction time on oxygen content of pickling niobium powder at $1133 \mathrm{~K}$

It can be seen from Table 6 and Figure 6 that when magnesium reduction temperature is $1133 \mathrm{~K}$, magnesium addition is $400 \%$ and reduction time is $2 \mathrm{~h}$, the oxygen content of pickling niobium powder is $445 \mathrm{ppm}$; when reduction time is $4 \mathrm{~h}$, the oxygen content of pickling niobium powder is $356 \mathrm{ppm}$; when reduction time is $5 \mathrm{~h}$ and $6 \mathrm{~h}$, the oxygen content of pickling niobium powder is $360 \mathrm{ppm}$ and $357 \mathrm{ppm}$ respectively. Therefore, when the reduction temperature is $1133 \mathrm{~K}$ and the amount of magnesium is $400 \%$, the oxygen content of pickling niobium powder decreases first and then remains unchanged when the reduction time increases from $2 \mathrm{~h}$ to $6 \mathrm{~h}$. The reason for the decrease is that with the extension of reduction time, the magnesium reduction reaction becomes more and more sufficient. When the reduction temperature increases from $1093 \mathrm{~K}$ to $1133 \mathrm{~K}$, the oxygen content of the acid washed niobium powder decreases from $416 \mathrm{ppm}$ to $356 \mathrm{ppm}$, and the reduction time is $1 \mathrm{~h}$ shorter than that corresponding to $1093 \mathrm{~K}$. The oxygen content of pickling niobium powder is obviously lower than that of pickling niobium powder corresponding to $200 \%$ and $300 \%$ magnesium addition when the magnesium addition is $400 \%$. When the magnesium addition is increased to $500 \%$ and $600 \%$, the oxygen content of pickling niobium powder is basically unchanged and the reduction product is 
prone to caking. Under the condition of $1133 \mathrm{~K}$ reduction temperature, the optimum magnesium reduction process parameters are as follows: $400 \%$ excess magnesium addition, $4 \mathrm{~h}$ reduction time and $356 \mathrm{ppm}$ oxygen content.

From the above effects of magnesium reduction temperature of $953 \mathrm{~K}, 1053$ $\mathrm{K}, 1093 \mathrm{~K}$ and $1133 \mathrm{~K}$ on the oxygen content of pickling niobium powder, it is known that when the reduction temperature increases from $953 \mathrm{~K}$ to $1133 \mathrm{~K}$, the oxygen content of pickling niobium powder decreases from $890 \mathrm{ppm}$ to $356 \mathrm{ppm}$, because with the increase of reduction temperature, the magnesium thermal reaction rate accelerates and the reaction becomes more and more sufficient. Continue to increase the reduction temperature, whether the oxygen content will continue to decrease? Continue to explore the effect of reduction temperature $1203 \mathrm{~K}$ on the oxygen content of pickling niobium powder, and the results are shown in Table 7 and Figure 7.

Table 7 Oxygen content of pickling niobium powder at $1203 \mathrm{~K}$ with different magnesium addition and reduction time (PPM)

\begin{tabular}{ccccc}
\hline Magnesium addition $/ \%$ & $\begin{array}{c}\text { Original niobium } \\
\text { powder }\end{array}$ & 300 & 400 & 600 \\
\hline Restore time $/ \mathrm{h}$ & 4100 & 462 & 431 & 419 \\
2 & 4100 & 489 & 440 & 426 \\
4 & 4100 & 551 & 455 & 442 \\
\hline
\end{tabular}

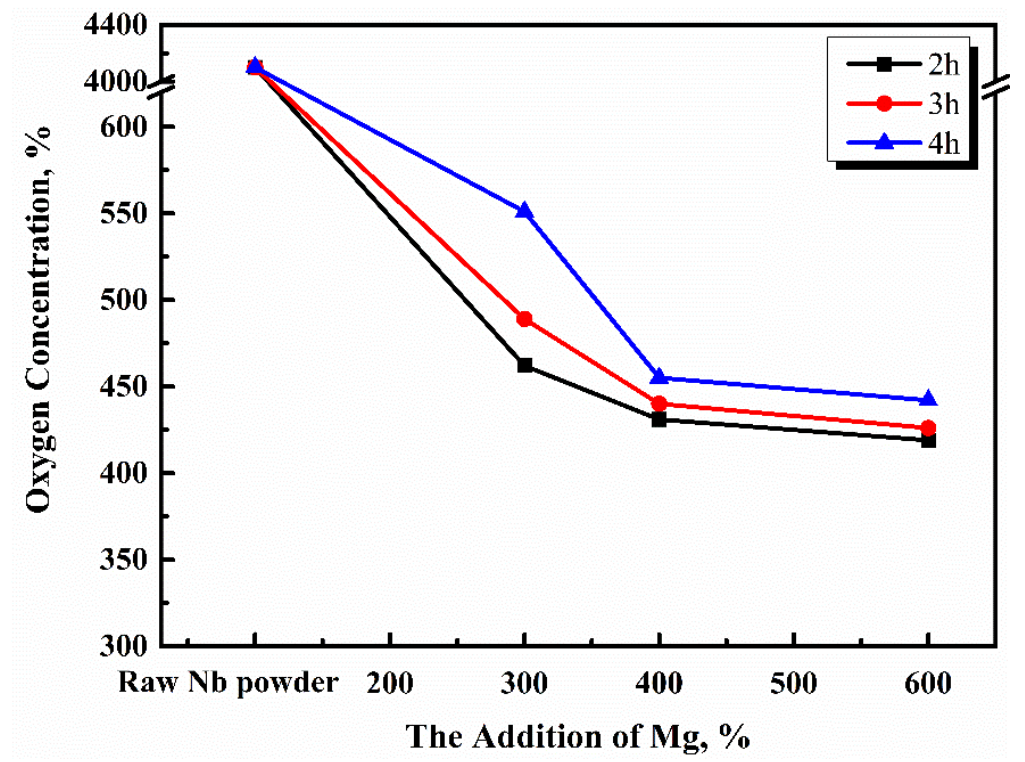


Figure 7 Effect of magnesium addition and reduction time at $1203 \mathrm{~K}$ on oxygen content of pickling niobium powder

It can be seen from Table 7 and Figure 7 that the oxygen content of pickling niobium powder corresponding to $300 \%, 400 \%$ and $600 \%$ magnesium addition is $462 \mathrm{ppm}, 431 \mathrm{ppm}$ and $419 \mathrm{ppm}$ respectively at $1203 \mathrm{~K}$ reduction for $2 \mathrm{~h}$; when the reduction temperature is increased to $3 \mathrm{~h}$, the oxygen content of pickling niobium powder corresponding to $300 \%, 400 \%$ and $600 \%$ magnesium addition is increased to $489 \mathrm{ppm}, 440 \mathrm{ppm}$ and $426 \mathrm{ppm}$ respectively; the reduction temperature is further increased to $4 \mathrm{~h}$. The oxygen content of pickling niobium powder increased to $551 \mathrm{ppm}, 455 \mathrm{ppm}$ and $442 \mathrm{ppm}$ respectively. It can be seen that the oxygen content of pickling niobium powder at $1203 \mathrm{~K}$ is generally higher than that at $1133 \mathrm{~K}$. Although the addition of magnesium increased from $300 \%$ to $600 \%$, the oxygen content of pickling niobium powder decreased, but the reduction products with excess magnesium of $400 \%, 500 \%$ and $600 \%$ had serious caking phenomenon, and the hardness was relatively large. A large amount of magnesium oxide and magnesium could be seen, which brought inconvenience to the subsequent pickling process. It can be seen that the high reduction temperature at $1203 \mathrm{~K}$ is not conducive to the reduction of oxygen content in pickling niobium powder. Therefore, the reduction process is optimized as follows: $1133 \mathrm{~K}$ reduction temperature, $400 \%$ excess magnesium addition for $4 \mathrm{~h}$, and the oxygen content of acid washed niobium powder is as low as $356 \mathrm{ppm}$. 


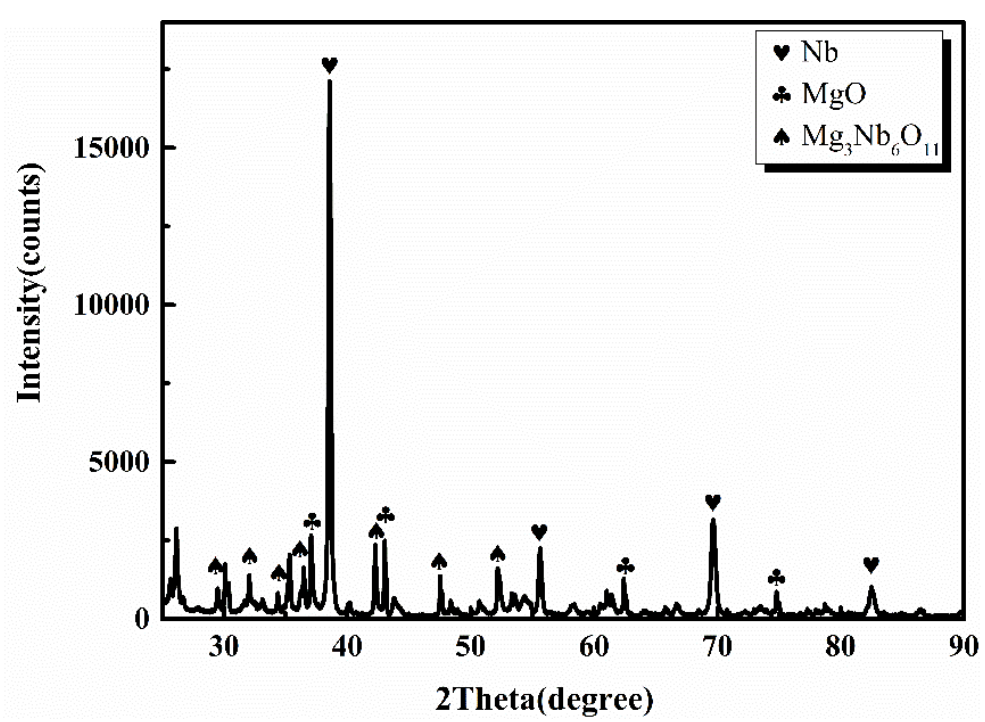

Figure 8 XRD pattern of reduction products of magnesium at $1203 \mathrm{~K}$ for $2 \mathrm{~h}$

It can be seen from Figure 8 that magnesium niobate $\mathrm{mg} 3 \mathrm{nbo11}$ is formed after reduction at $1203 \mathrm{~K}$ for $2 \mathrm{~h}$. As a result, the oxygen content of $1203 \mathrm{~K}$ reduced niobium powder after pickling is higher than that of $1133 \mathrm{~K}$ reduced niobium powder [24-26]. It is known from that reduction temperature, reduction time and magnesium addition all have great influence on oxygen content of pickling niobium powder. With the increase of reduction temperature from $953 \mathrm{~K}$ to $1133 \mathrm{~K}$, the oxygen content of pickling niobium powder decreases from $890 \mathrm{ppm}$ to $356 \mathrm{ppm}$, which is due to the increase of reduction temperature, the reduction rate of magnesium is accelerated, and the reduction reaction is more and more sufficient; with the increase of reduction temperature to $1203 \mathrm{~K}$, the oxygen content of pickling niobium powder increases, which is due to the presence of acid insoluble magnesium niobate in the reduction product.

The effect of reduction time on oxygen content of pickling niobium powder was studied under the condition of sufficient magnesium addition. At 
$1053 \mathrm{~K}$ for $2-8 \mathrm{~h}$, the oxygen content of pickled niobium powder changed little, about $530 \mathrm{ppm}$; at $1093 \mathrm{~K}$ and $400 \% \mathrm{Mg}$ for 2-6 h, the oxygen content of pickled niobium powder decreased from $481 \mathrm{ppm}$ in $2 \mathrm{~h}$ to $406 \mathrm{ppm}$ in 5 $\mathrm{h}$; at $1133 \mathrm{~K}$ for $6 \mathrm{~h}$, the oxygen content of pickled niobium powder remained unchanged, about 403 ppm K. The oxygen content of acid washed niobium powder decreased from $445 \mathrm{ppm}$ in $2 \mathrm{~h}$ to $356 \mathrm{ppm}$ in $4 \mathrm{~h}$ after $2-6 \mathrm{~h}$ reduction with $400 \% \mathrm{Mg}$ Addition. The oxygen content of acid washed niobium powder changed from $356 \mathrm{ppm}$ to $357 \mathrm{ppm}$ after $6 \mathrm{~h}$ reduction with increasing reduction time. Therefore, at the reduction temperature of $1093 \mathrm{~K}$ and 1133 $\mathrm{K}$, the oxygen content of pickling niobium powder first decreases and then remains unchanged with the extension of reduction time.

At the same reduction temperature and time, the oxygen content of pickling niobium powder first decreases and then remains unchanged with the increase of magnesium content. The results show that the reduction products are relatively loose when the magnesium addition is $200 \%$ and $300 \%$ too much; when the magnesium addition is $400 \%$ too much, the reduction products obviously agglomerate, but can be broken with a little force, the reduction products are just loose, and the oxygen content of pickling niobium powder is the lowest; when the magnesium addition is $500 \%$, the reduction products agglomerate more, the hardness increases, and the oxygen content of pickling niobium powder is basically the same as that of $400 \%$ magnesium The addition amount is the same. Therefore, the optimal reduction process 
parameters of magnesium reduction were optimized as follows: reduction temperature $1133 \mathrm{~K}$, reduction time $4 \mathrm{~h}$, excess magnesium addition $400 \%$.

\subsection{Effect of reducing atmosphere and crucible material on oxygen content and} phase composition of pickling niobium powder

In order to explore the influence of reduction atmosphere and material of charging Crucible on oxygen content of acid washed niobium powder, the reduction process of $1133 \mathrm{~K}$ reduction temperature, $400 \%$ magnesium addition and $4 \mathrm{~h}$ reduction was adopted to study the influence of hydrogen, argon and vacuum reduction atmosphere, graphite crucible and Nickel Crucible on oxygen content of acid washed niobium powder. As shown in table 8, the oxygen content of acid washed niobium powder obtained by graphite crucible and Nickel Crucible in vacuum and argon reduction atmosphere is given respectively.

Table 8 Oxygen content (PPM) of acid washed niobium powder reduced by different reduction atmosphere and crucible

\begin{tabular}{ccc}
\hline Reducing atmosphere & Argon atmosphere \\
Crucible materiat & 764 & 371 \\
Nickel Crucible & 802 & 365 \\
Graphite crucible & Vacuum atmosphere & . \\
\hline
\end{tabular}

It can be seen from table 8 that the oxygen content of acid washed niobium powder obtained by Nickel Crucible and graphite crucible charging reduction in argon state is basically the same, about $365 \mathrm{ppm}$. But in vacuum, the oxygen content of niobium powder is 764 ppm in Nickel Crucible and 802 ppm in graphite crucible. The oxygen content of niobium powder by vacuum reduction and pickling with two kinds of crucible is higher than that of argon protection. The main reasons are as follows: first, there will inevitably be air infiltration in the hydrogenation and dehydrogenation furnace in the vacuum state. In the process of vacuum cooling, when the cooling temperature is $573{ }^{\circ} \mathrm{C}$ Above $\mathrm{K}$, the surface of niobium powder with high activity will be oxidized to niobium oxide again, so niobium powder will be oxidized again after oxygen reduction; 
secondly, with the volatilization of magnesium in vacuum and the decrease of temperature in the process of cooling, the oxygen reduction ability of niobium powder gradually decreases. However, in the high purity argon environment, the pressure in the furnace has been maintained at about $0.1 \mathrm{MPa}$, and the outside air is not easy to enter the furnace, so it is easier to obtain niobium powder with lower oxygen content in argon atmosphere than in vacuum.

The reason why the oxygen reduction effect of niobium powder using Nickel Crucible in vacuum is better than that using graphite crucible is that the nickel crucible has a cover in the reduction process, and the magnesium vapor always fills the whole Nickel Crucible; while the graphite crucible has no cover, the magnesium vapor gradually volatilizes faster, which is not conducive to the full reduction of niobium powder.

Niobium powder has good hydrogen absorption properties, and can easily react with hydrogen to form brittle niobium hydride. Niobium has the fastest formation rate of $\mathrm{NbH}$ at about $633 \mathrm{~K}$, and $\mathrm{NbH}$ begins to dehydrogenate at about $923 \mathrm{~K}$. In order to investigate the hydrogen absorption of niobium powder in the reduction process in hydrogen atmosphere, the reduction experiment of niobium powder in $1133 \mathrm{~K}$ and hydrogen atmosphere was carried out, and the reduction products were analyzed by XRD diffraction. The results are shown in Figure 9.

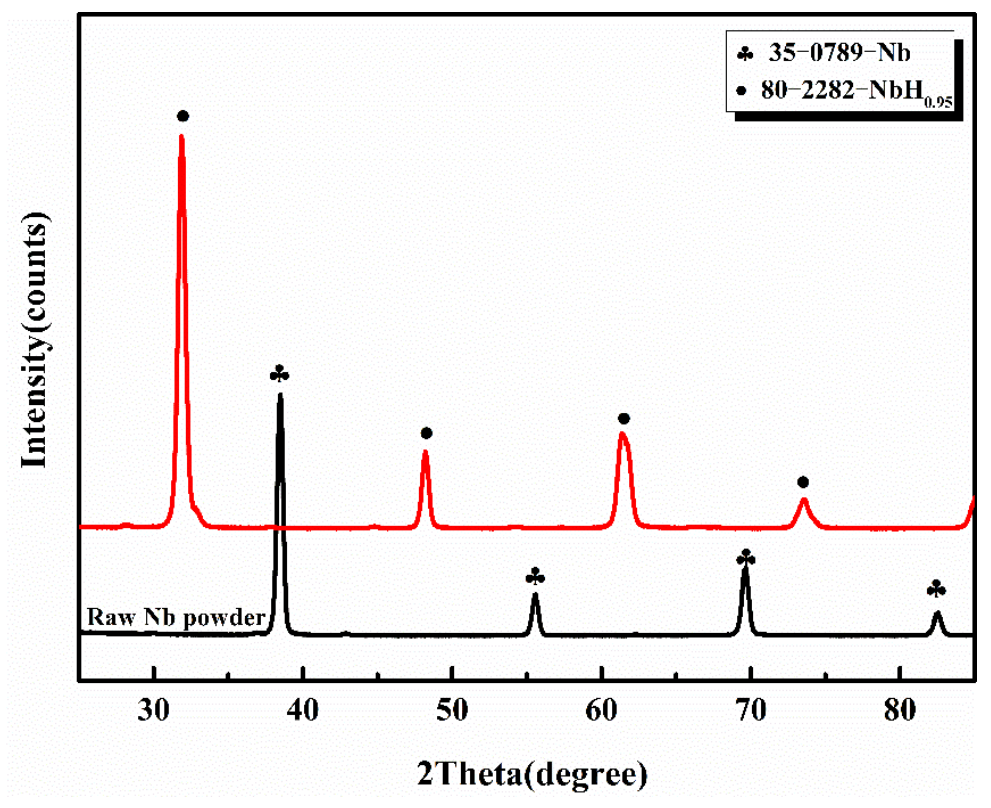


Figure $9 \mathrm{XRD}$ diffraction pattern of $\mathrm{Nb}$ powder reduced in hydrogen medium

It can be seen from figure 9 that in hydrogen atmosphere, the diffraction peak position of niobium powder after magnesium reduction is consistent with that of 80-2282-nbh0.95, indicating that niobium powder is completely transformed into nbh0.95 after reduction in hydrogen atmosphere. Therefore, it is not feasible to reduce niobium powder in hydrogen state. At high temperature, nickel is easy to form low melting point eutectic with magnesium, resulting in the increase of nickel content in pickling niobium powder. The carbon content of niobium powder can be increased by $70 \mathrm{ppm}$ when carbon crucible is used. Therefore, it is necessary to re select the crucible material in the future research.

3.4 Effect of oxygen reduction by magnesium on particle size, morphology and solid solution Oxygen of niobium powder

In order to investigate the effect of reduction and pickling on the particle size and morphology of niobium powder. The particle size and morphology of the original niobium powder with high oxygen and the original niobium powder were studied at $1133 \mathrm{~K}$ reduction temperature, $400 \%$ magnesium addition and 4 $\mathrm{h}$ reduction. Figure 10 is the SEM micrograph of original niobium powder and oxygen reducing niobium powder, and Figure 11 is the particle size distribution of niobium powder before and after oxygen reducing. 

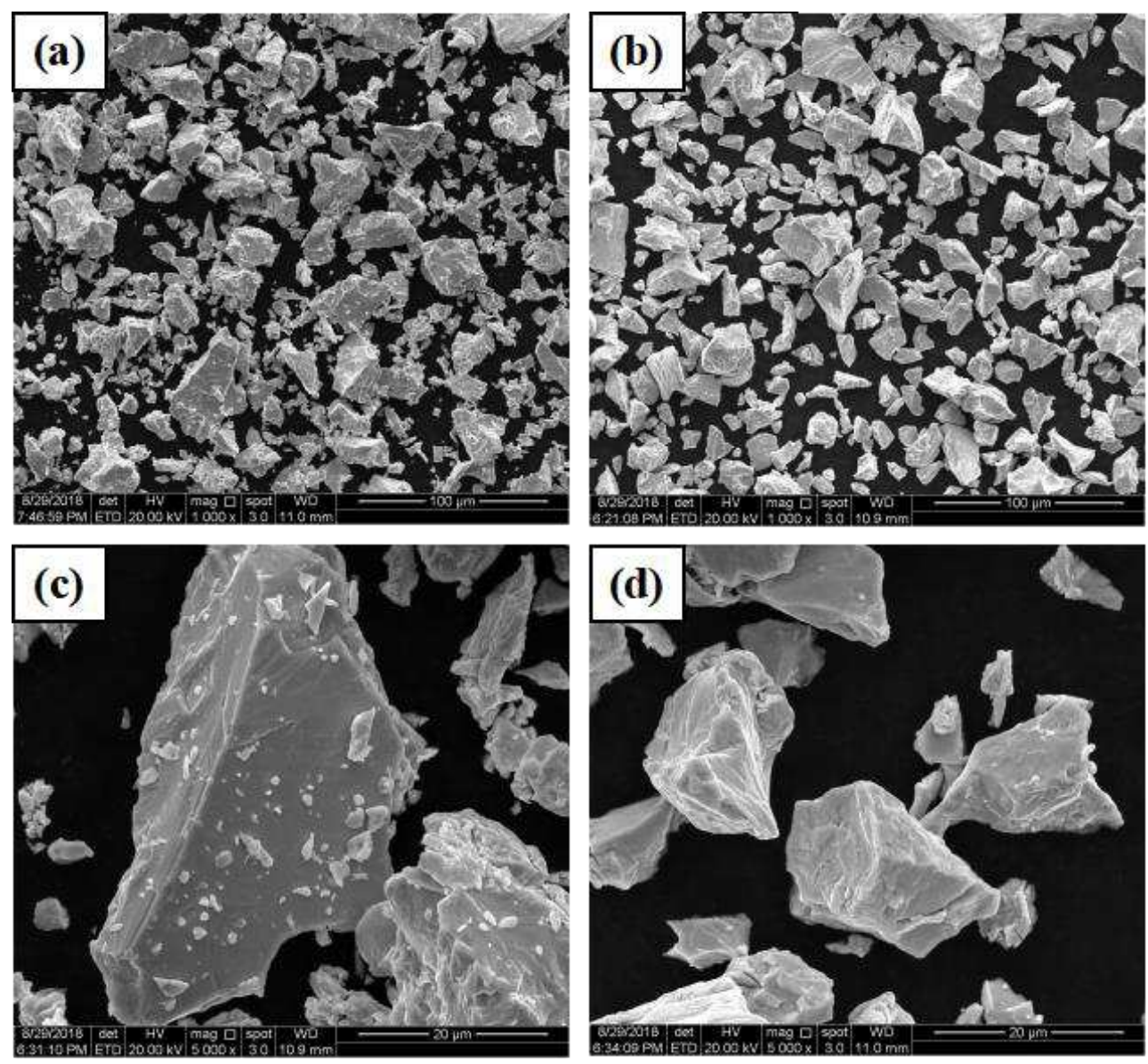

Figure 10 SEM pictures of niobium powder before and after oxygen reduction:

(a) low rate before oxygen reduction, (b) low rate after oxygen reduction, (c) high rate before oxygen reduction, (d) high rate after oxygen reduction

It can be seen from Figure 10 (a) that the larger particle size of niobium powder before oxygen reduction is about $40 \mathrm{um}$, and the smaller one is about $0.5 \mathrm{um}$. It can be seen from Figure 10 (c) that the shape of niobium powder before oxygen reduction is irregular and sharp, and there are many fine particles about 0.2-2 um adsorbed on the surface of large particles. It can be seen from figure 10 (b) that the minimum particle size of niobium powder after oxygen reduction is about 5 um, and the maximum particle size is still about $40 \mathrm{um}$, with no obvious change in morphology compared with that before deoxidation. Therefore, in the process of reducing and pickling, the oxygen reduction of niobium powder basically does not change the morphology of niobium powder, but it can be seen from Figure 10 (d) that the niobium powder of fine particles is greatly reduced after oxygen reduction, and it can be seen that the fine particles are dissolved or washed away 
by acid in the process of pickling and water washing. The average particle size of original niobium powder and niobium powder after oxygen reduction are 9.8 um and 10.2 um respectively; the particle size distribution of niobium powder before and after oxygen reduction is shown in Figure 11.

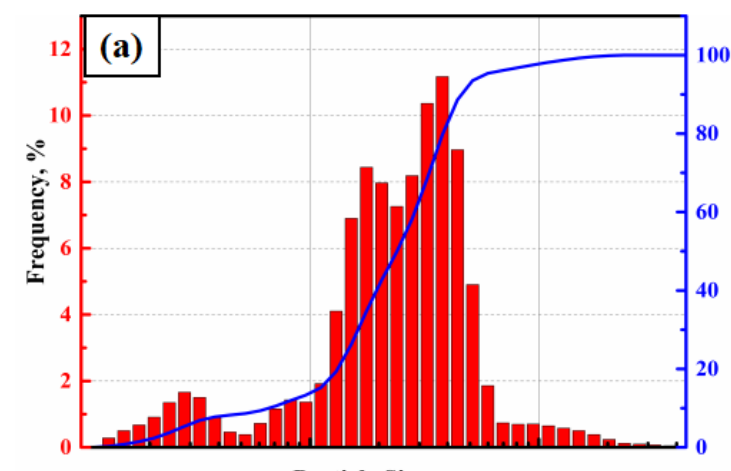

Particle Size, um

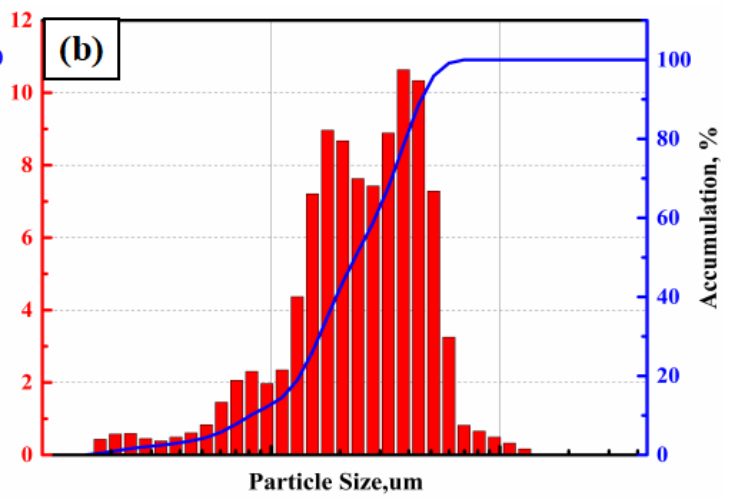

Particle Size,um

Figure 11 particle size distribution of niobium powder before and after oxygen reduction: (a)

$$
\text { before pickling, (b) after pickling }
$$

It can be seen from figure 11 that the oxygen reduction treatment causes the loss of very fine particles in niobium powder. According to calculation, the loss rate of niobium powder after oxygen reduction treatment is about $0.3 \%$.

In order to explore whether oxygen reduction treatment will affect the dissolved oxygen in niobium powder. XRD analysis was carried out on the acid washed niobium powder at $1133 \mathrm{~K}$ reduction temperature, $400 \%$ magnesium addition and reduction for $4 \mathrm{~h}$. the diffraction pattern is shown in Figure 12.

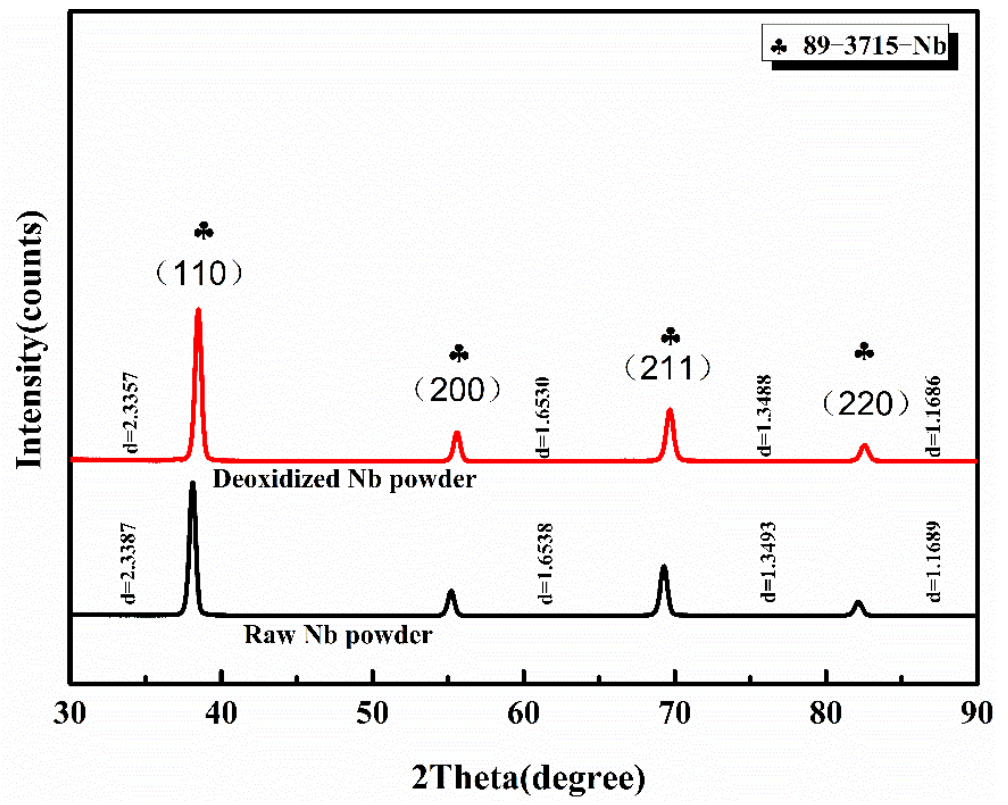


Figure 12 XRD diffraction pattern of niobium powder before and after deoxidization

It can be seen from Figure 12 that there is no new phase formed after acid pickling of $\mathrm{Nb}$ powder reduced for $4 \mathrm{~h}$ at $1133 \mathrm{~K}$ reduction temperature and $400 \%$ $\mathrm{Mg}$ addition, but only a single NB phase. The peak shape of the diffraction peak is basically unchanged without obvious broadening. However, the crystal plane spacing of niobium powder (110), (200), (211), (220) before oxygen reduction tends to decrease compared with that before oxygen reduction. For example, the (110) plane spacing D decreases from $2.3387 \mathrm{~nm}$ before oxygen reduction to $2.3357 \mathrm{~nm}$ after oxygen reduction, and the peak position angle shifts to the right by about $0.8^{\circ} \mathrm{C}$. It can be seen that oxygen reduction not only reduces the surface oxygen of niobium powder, but also reduces the solid dissolved oxygen. This is due to the lattice distortion of niobium caused by oxygen dissolved in the niobium lattice. With the decrease of oxygen content in niobium powder, the degree of solid solution of oxygen in niobium decreases, which leads to the decrease of lattice distortion, that is, the decrease of crystal plane spacing D. From the Bragg equation,

$$
2 d \sin \theta=n \lambda
$$

( $\mathrm{N}$ and $\lambda$ are the reflection order and $\mathrm{X}$-ray wavelength respectively, $\mathrm{D}$ is the crystal plane spacing, and $\theta$ is the angle between the incident ray or reflected ray and the reflecting surface) When $\mathrm{D}$ decreases, $\theta$ increases. Therefore, the peak position of $\mathrm{Nb}$ powder after oxygen reduction treatment moves to the right relative to the original NB powder.

\section{Conclusion}

In this paper, the effects of magnesium addition, reduction temperature, reduction time, crucible material and reduction atmosphere on the oxygen content of niobium powder after pickling were studied. The main conclusions are as follows: Reduction temperature, magnesium addition and reduction time 
all have great influence on the oxygen content of acid washed niobium powder. When the reduction temperature increases from $953 \mathrm{~K}$ to $1133 \mathrm{~K}$, the oxygen content of niobium powder decreases from $890 \mathrm{ppm}$ to $356 \mathrm{ppm}$. When the reduction temperature increases to $1203 \mathrm{~K}$, the oxygen content of niobium powder increases. When the amount of magnesium is enough, the effect of reduction time on niobium powder is different with the reduction temperature. When the reduction temperature is $1053 \mathrm{~K}$ and the reduction time is $2-8 \mathrm{~h}$, the oxygen content of pickled niobium powder changes little, about 530 ppm; when the reduction time is $2-6 \mathrm{~h}$ at $1093 \mathrm{~K}$ and $1133 \mathrm{~K}$, the oxygen content of pickled niobium powder first decreases and then remains unchanged, from $481 \mathrm{ppm}$ to $406 \mathrm{ppm}$ and from $445 \mathrm{ppm}$ to $357 \mathrm{ppm}$, respectively. At the same reduction temperature and time, the oxygen content of pickling niobium powder gradually decreases to a certain value with the increase of magnesium addition, and remains unchanged. In addition, in argon atmosphere and graphite crucible, the oxygen content of niobium powder obtained by oxygen reduction is better than that in vacuum atmosphere. The average particle size and morphology of niobium powder after oxygen reduction were observed. Compared with that before oxygen reduction, there was almost no change in niobium powder, but a small amount of fine niobium powder was removed. After oxygen reduction, the peak angle of niobium powder shifts to the right by about $0.8^{\circ} \mathrm{C}$, resulting in the decrease of crystal plane spacing. Finally, when the reduction temperature is $1133 \mathrm{~K}$, the excess amount of magnesium is $400 \%$, and the reduction time is 4 $\mathrm{h}$, the oxygen content of the pickling niobium powder is reduced from $4100 \mathrm{ppm}$ to $356 \mathrm{ppm}$, which greatly reduces the oxygen content of the industrial niobium powder.

\section{Declaration of interests:}

The authors declare that they have no known competing financial interests or personal relationships that could have appeared to influence the work reported 
in this paper.

\section{Reference:}

[1] Eckert J. Niobium and Niobium Compounds[M]. Ullmann's Encyclopedia of Industrial Chemistry, 2000

[2] Florêncio O, Silva P S, Grandini C R. Diffusion Phenomena of the Oxygen and Nitrogen in Niobium by Mechanical Spectroscopy[J]. Defect \& Diffusion Forum, 2010, 297-301: 1346-1353

[3] Yamanaka S , Fujita Y, Uno M , et al. Influence of interstitial oxygen on hydrogen solubility in metals[J]. Journal of Alloys and Compounds, 1999, 293-295(none): 0-51

[4] Trivisonno J, Washick J, Keck M J, et al. Ultrasonic attenuation of longitudinal and shear waves in the normal and superconducting states of niobium[J]. Journal of Low Temperature Physics, 1971, 4(1): 97-109

[5] Parraud S, Hubert P, falzgraf L G, et al. Stabilization and Characterization of Nanosized Niobium and Niobium Oxide Sols-Optical Applications for High-Power Lasers[J]. Journal of the American Ceramic Society, 75(8): 4

[6] Okabe T H, Iwata S, Imagunbai M, et al. Production of niobium powder by preform reduction process using various fluxes and alloy reductant[J]. ISIJ International [0915-1559] Iwata, 2004, 44(2): 285-293

[7] Yuan B, Okabe T H. Production of fine niobium powder by preform reduction process using $\mathrm{Mg}-\mathrm{Ag}$ alloy reductant[J]. Cheminform, 2007, 443(1-2): 0-80

[8] Orlov V M, Sukhorukov V V. Magnesium-thermal preparation of niobium powders[J]. Russian Metallurgy, 2010, 2010(3): 168-173

[9] T Satish Kumar. Preparation of Niobium Metal Powder by Two-Stage Magnesium Vapor Reduction of Niobium Pentoxide[J]. Hindawi Publishing Corporation Journal of Metallurgy, 2013, 80: $1-8$

[10] Nico C, Monteiro T, M.P.F. Graça. Niobium Oxides and Niobates physical properties: review and prospects[J]. Progress in Materials Science, 2016, 80: 1-37

[11] Naito K, Matsui T. Review on phase equilibria and defect structures in the niobium-oxygen system[J]. Solid State Ionics, 1984, 12(none): 125-134

[12] Wu A T. Investigation of oxide layer structure on niobium surface using a secondary ion mass spectrometry[J]. Physica C, 2006, 441(1-2): 79-82

[13] Hong C I, Lim J W. Efficacy of Acid Cleaning on the Deoxidation of Titanium Powder Using Calcium [J]. Korean Journal Of Metals And Materials, 2018, 56(3): 205-209

[14] Lai H, Huang L, Gan C, et al. Enhanced acid leaching of metallurgical grade silicon in hydrofluoric acid containing hydrogen peroxide as oxidizing agent[J]. Hydrometallurgy, 2016, 164 : $103-110$

[15] Salih Aydoğan, Aras A, Gökhan Uçar, et al. Dissolution kinetics of galena in acetic acid solutions with hydrogen peroxide[J]. Hydrometallurgy, 2007, 89(3): 189-195

[16] Lu D L, Hu Y Y, Lin T, et al. Refining of Metallurgical Grade Silicon by Acid Leaching[J]. Advanced Materials Research, 2010, 156-157: 566-569

[17] Xie K, Mai Y, Ma W, et al. The Removal of Titanium from Metallurgical Silicon by HF-HCL Leaching[J]. Metallurgist, 2013, 57(7-8): 633-638 
[18] Lai H, Huang L, Xiong H, et al. Hydrometallurgical Purification of Metallurgical Grade Silicon with Hydrogen Peroxide in Hydrofluoric Acid[J]. Industrial \& Engineering Chemistry Research, 2017, 56(1): 311-318

[19] Banchorndhevakul W, Matsui T, Naito K. Vaporization Study on Vanadium-Oxygen Solid Solution by Mass Spectrometric Method[J]. Journal of Nuclear Science and Technology, 1986, 23(10): 873-882

[20] Mueller R, Bobeth M, Brumm H, et al. Kinetics of nanoscale structure development during Mg-vapour reduction of Niobium oxide[J]. International journal of materials research [1862-5282] Bobeth, 2007, 98(11): 1138-1145

[21] Orlov V M, Kryzhanov M V. Deoxidation of the Niobium powder produced by selfpropagating high-temperature synthesis[J]. Russian Metallurgy, 2014, 2014(3): 191-194

[22] Livingston J D, Cahn J W. Discontinuous coarsening of aligned eutectoids[J]. Acta Metallurgica, 1974, 22(4): 495-503

[23] Kyunsuk Choi, Hanshin Choi. Effect of magnesium on the phase equilibria in magnesiothermic reduction of Nb2O5[J]. Materials Letters, 2016, 183: 151-155

[24] Pukazhselvan D, Otero-Irurueta G, Pérez J, et al. Crystal structure phase stoichiometry and chemical environment of MgxNbyOx+y nanoparticles and their impact on hydrogen storage in MgH2[J]. International Journal of Hydrogen Energy, 2016, 41(27): 1709-1715

[25] You Y C, Park H L, Song Y G, et al. Stable phases in the MgO-Nb2O5 system at $1250^{\circ} \mathrm{C}[\mathrm{J}]$. Journal of Materials Science Letters, 1994, 13: 1487-1489

\{26] Ananta S. Phase and morphology evolution of magnesium niobate powders synthesized by solid-state reaction[J]. Materials Letters, 2004, 58(22-23): 0-2786 
Figures

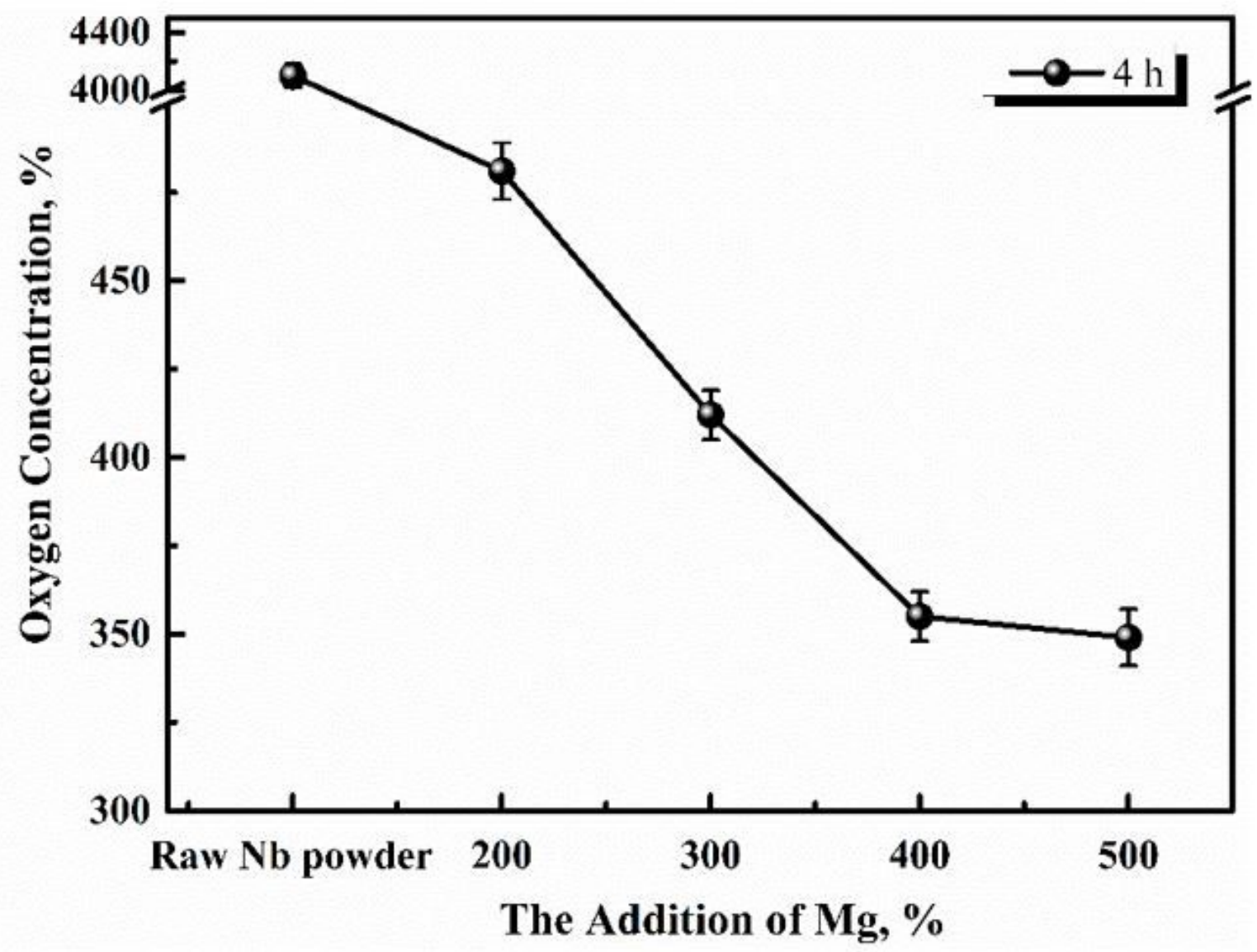

Figure 1

Effect of magnesium addition on oxygen content of pickling niobium powder at $1133 \mathrm{~K}$ for $4 \mathrm{~h}$ 

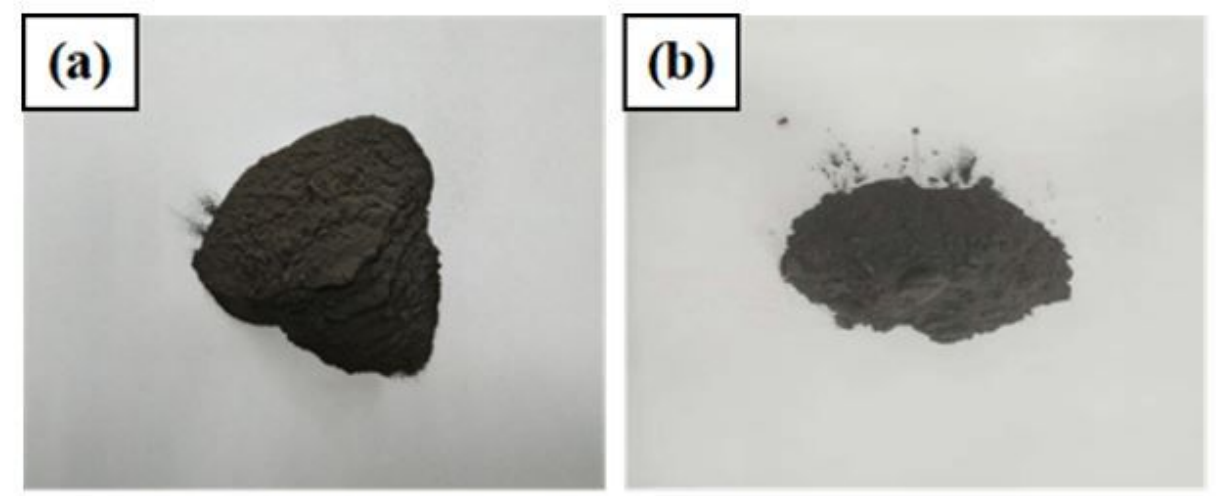

\section{(c)}
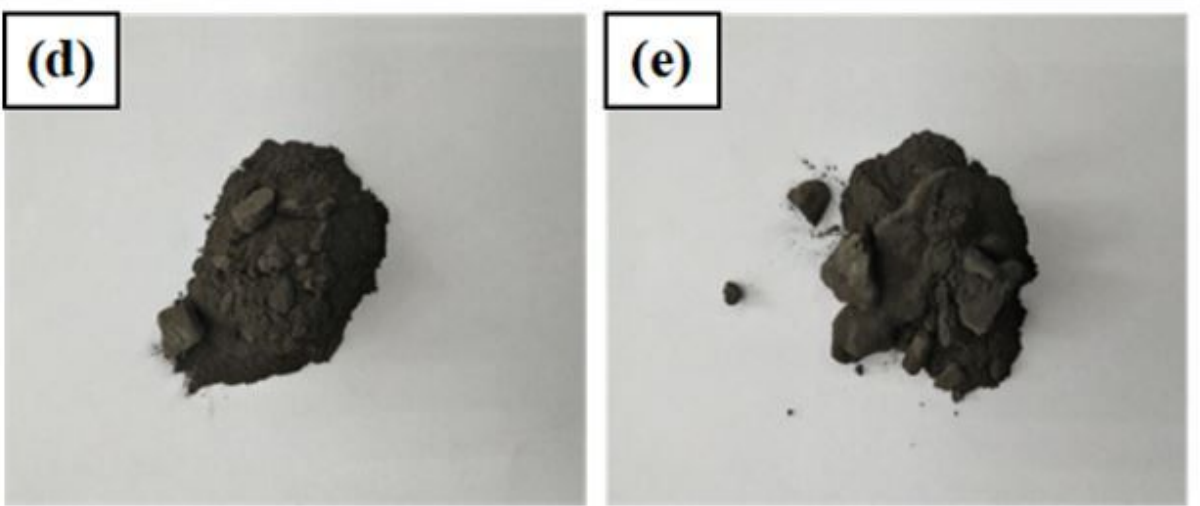

Figure 2

Different Mg Addition on morphology of reduced niobium powder 


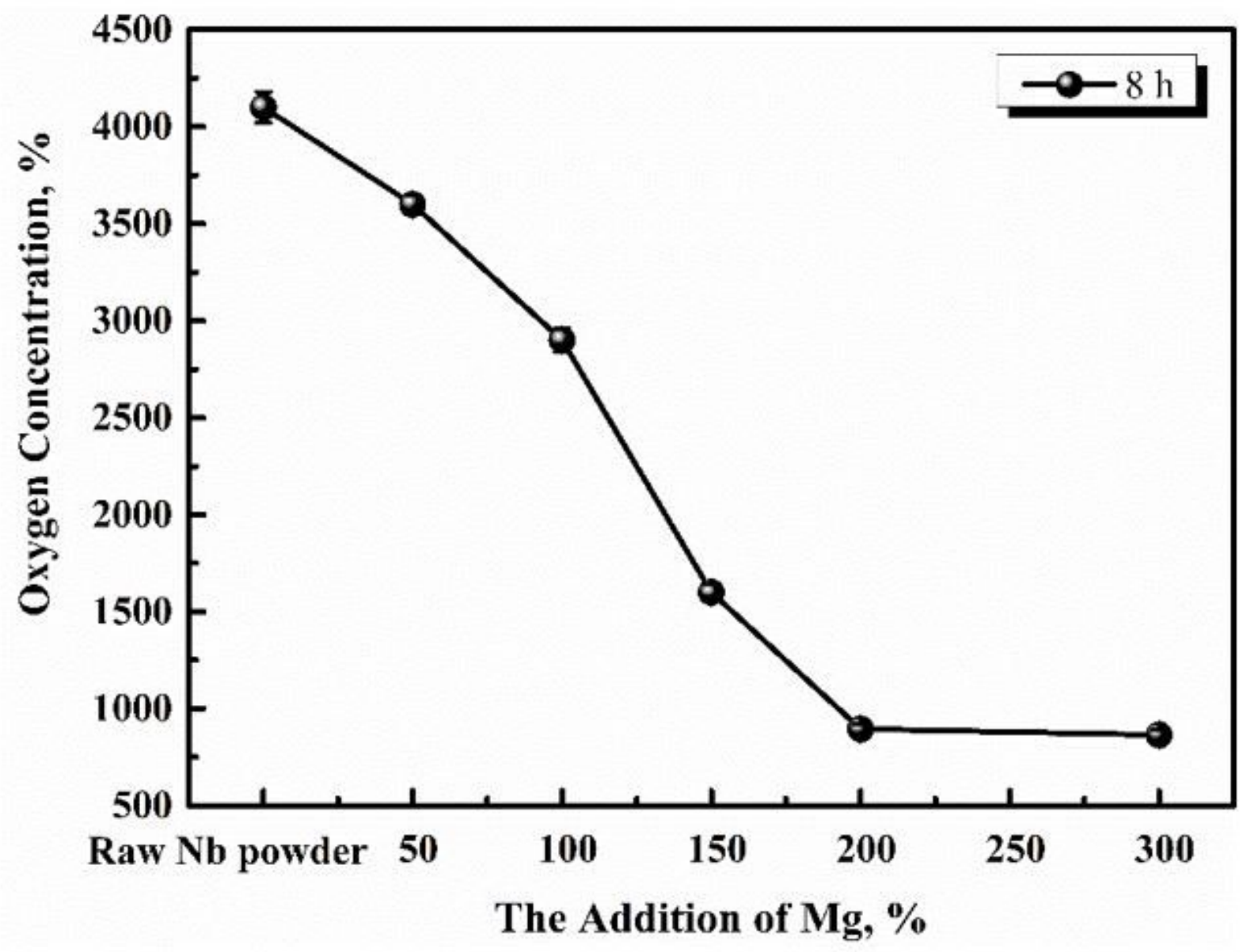

Figure 3

Oxygen content of pickling niobium powder at $953 \mathrm{~K}$ for $8 \mathrm{~h}$ with different magnesium addition 


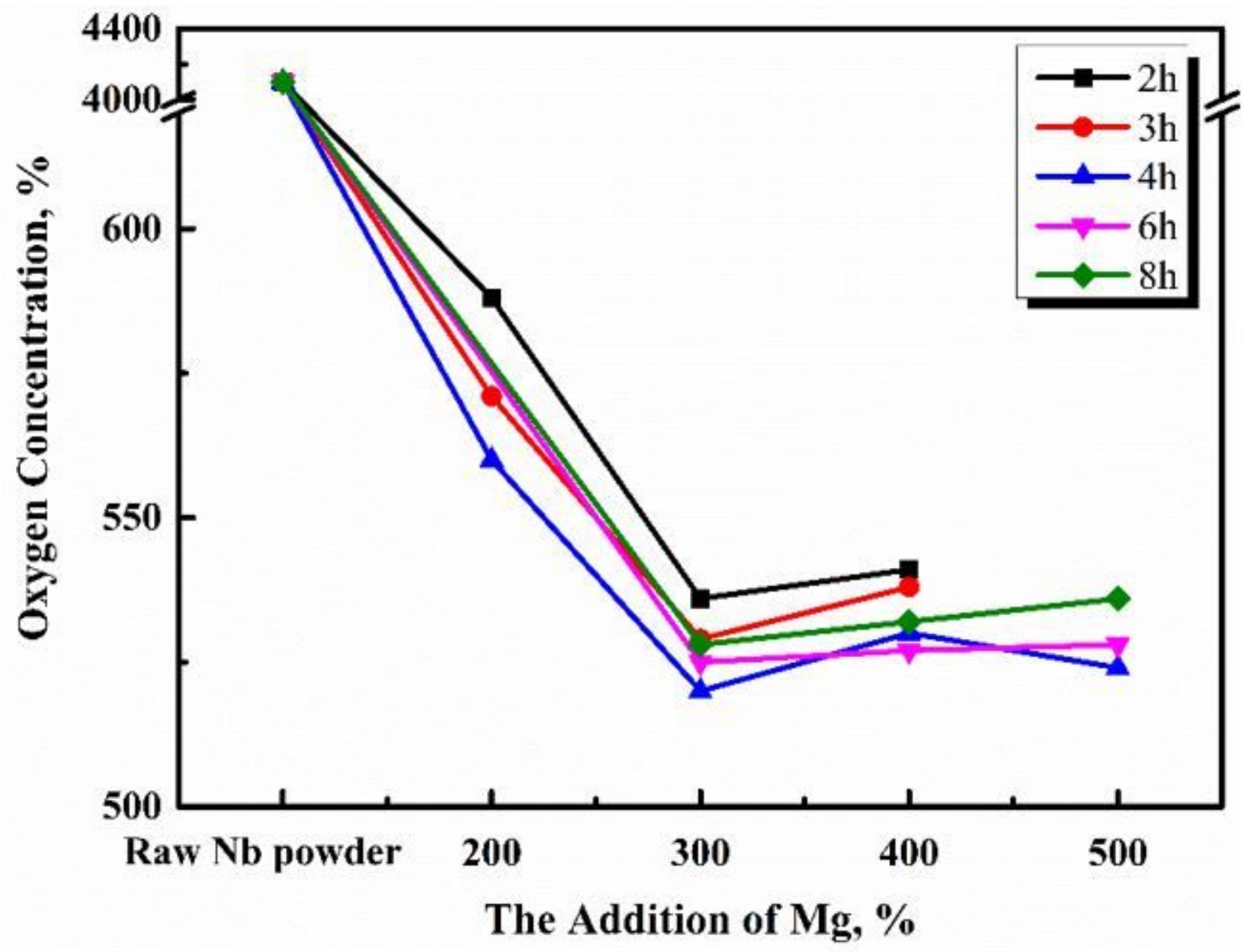

Figure 4

Effect of magnesium addition and reduction time on oxygen content of pickling niobium powder at 1053 $\mathrm{K}$ 


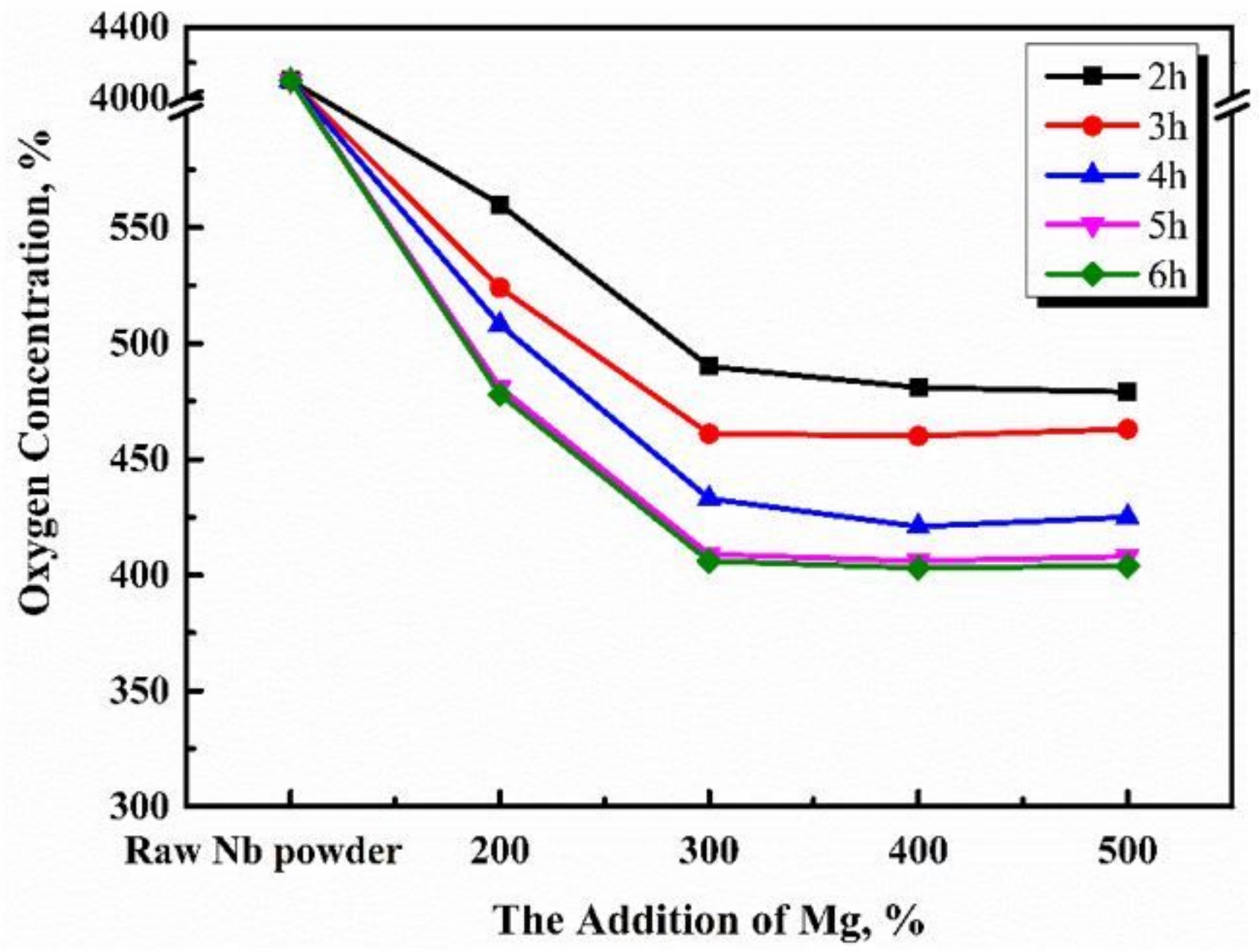

Figure 5

Effect of magnesium addition and reduction time on oxygen content of pickling niobium powder at 1093 $\mathrm{K}$ 


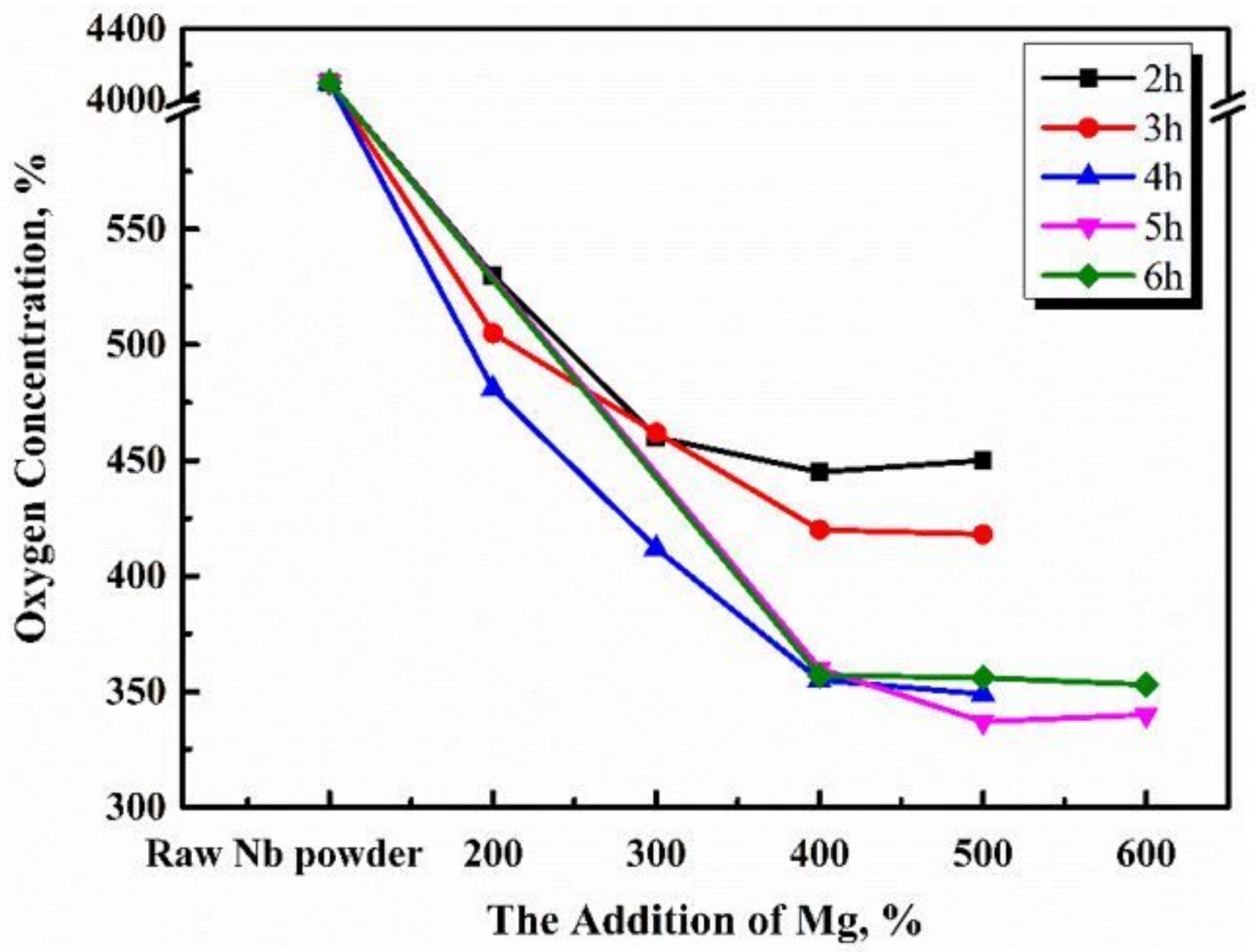

Figure 6

Effect of magnesium addition and reduction time on oxygen content of pickling niobium powder at 1133 $\mathrm{K}$ 


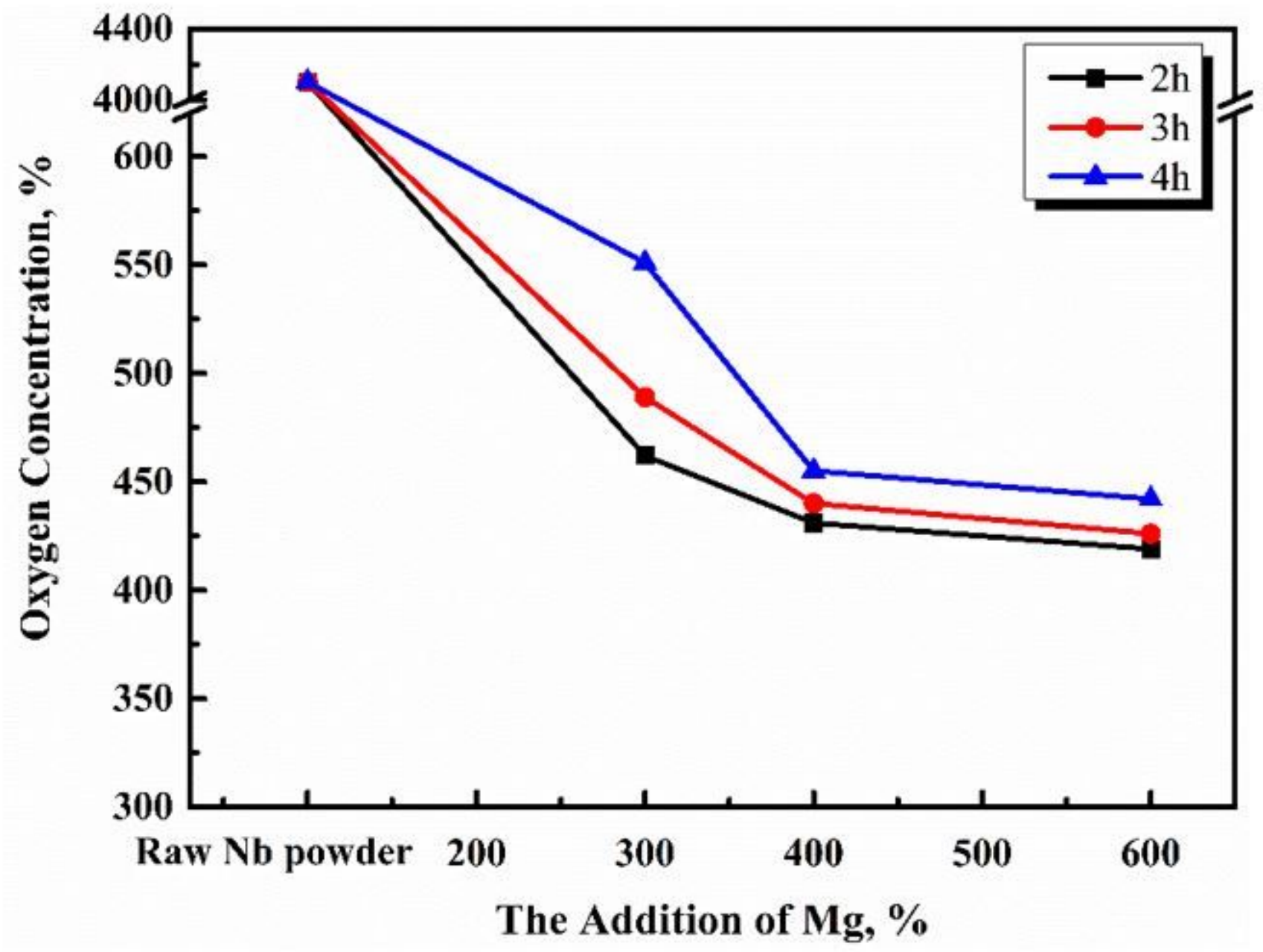

Figure 7

Effect of magnesium addition and reduction time at $1203 \mathrm{~K}$ on oxygen content of pickling niobium powder 


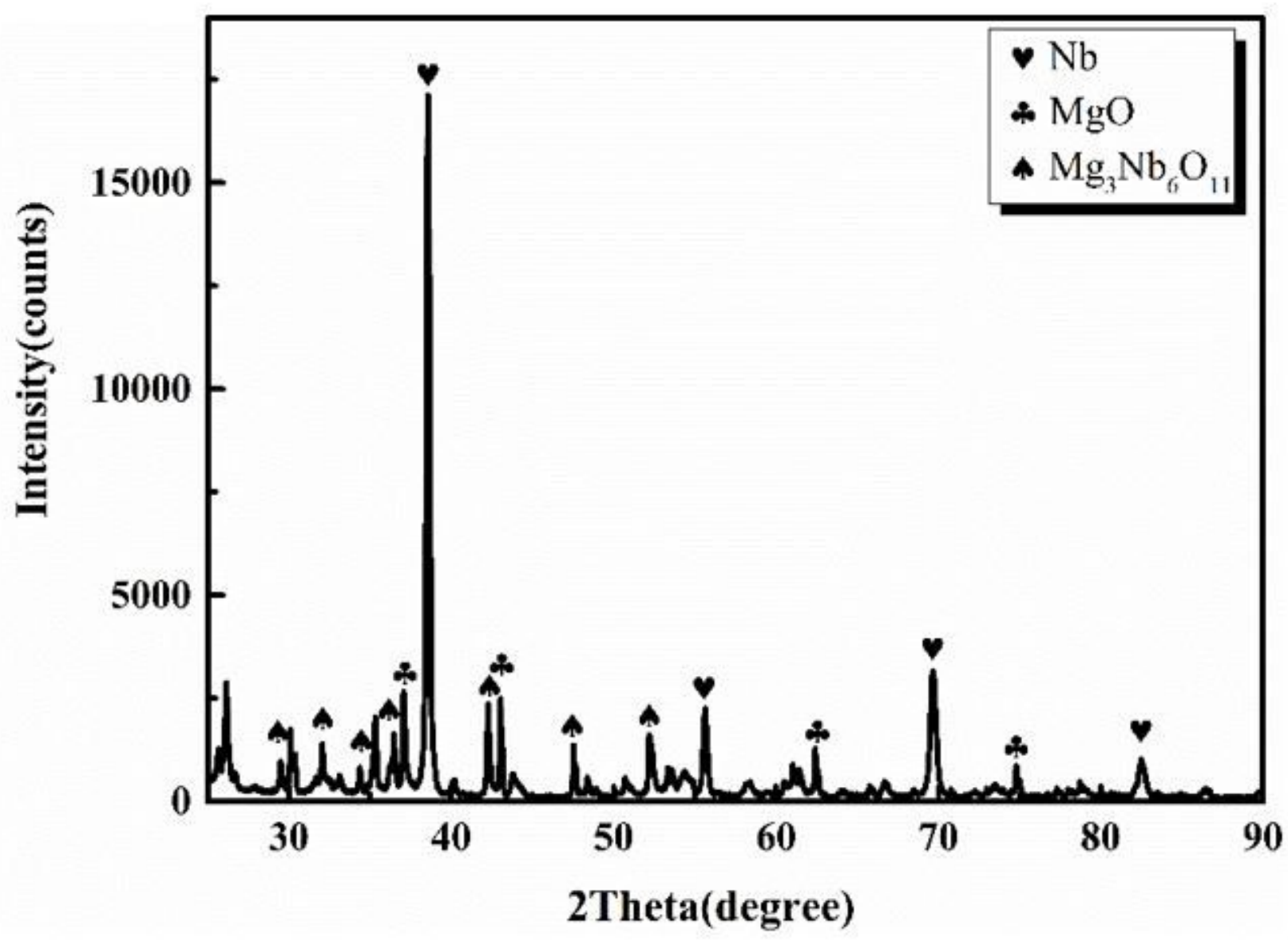

Figure 8

XRD pattern of reduction products of magnesium at $1203 \mathrm{~K}$ for $2 \mathrm{~h}$ 


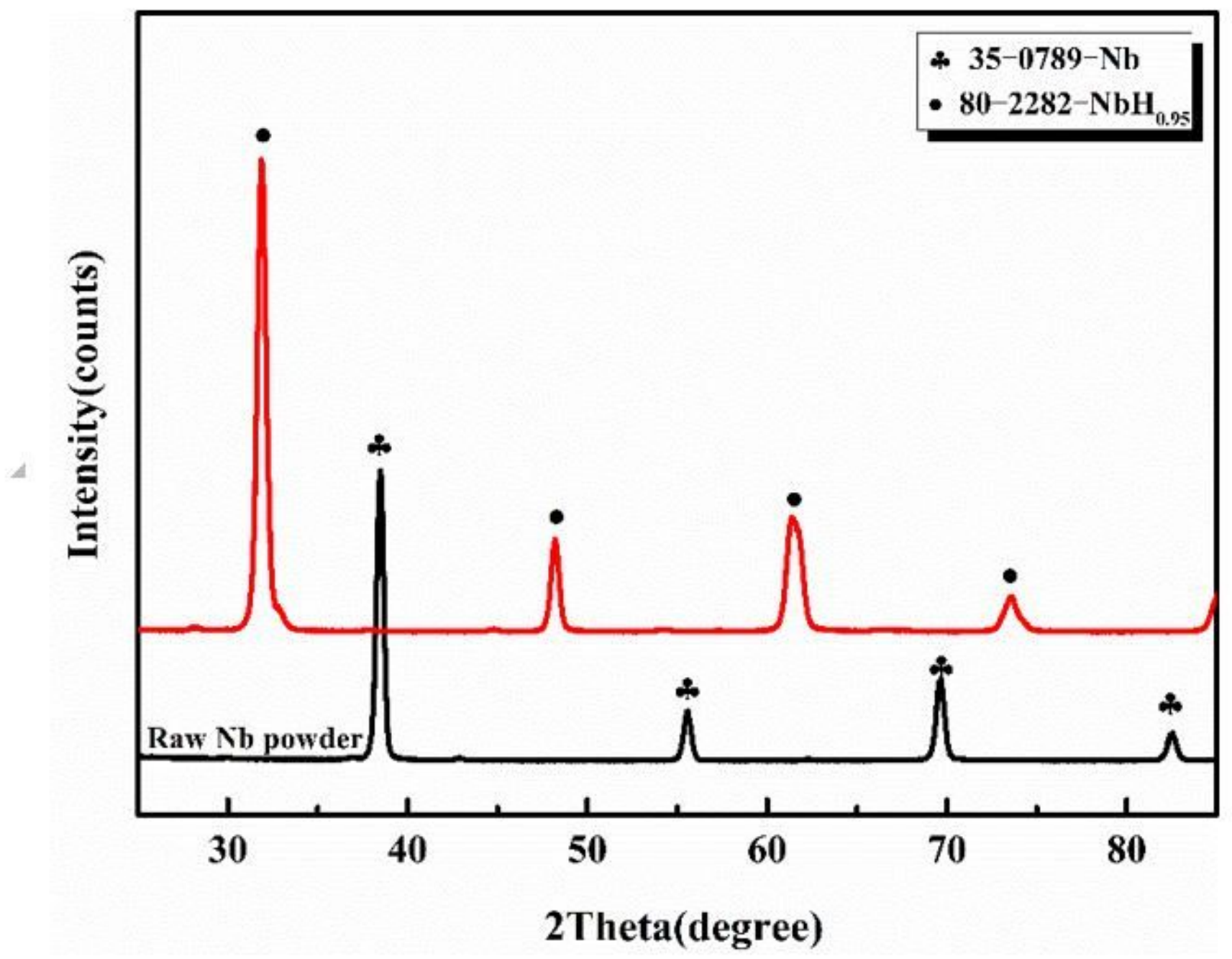

Figure 9

XRD diffraction pattern of $\mathrm{Nb}$ powder reduced in hydrogen medium 

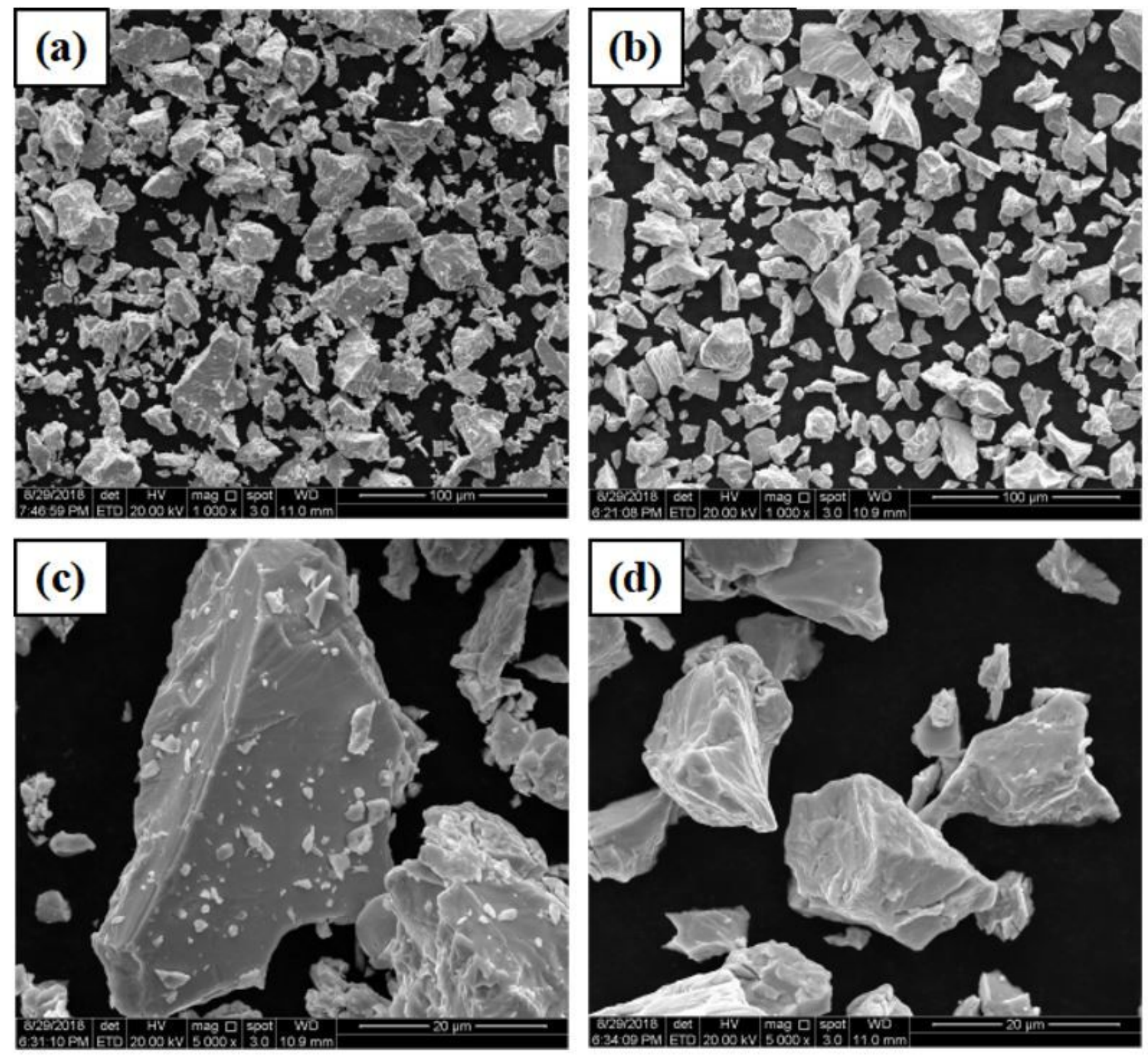

Figure 10

SEM pictures of niobium powder before and after oxygen reduction: (a) low rate before oxygen reduction, (b) low rate after oxygen reduction, (c) high rate before oxygen reduction, (d) high rate after oxygen reduction 


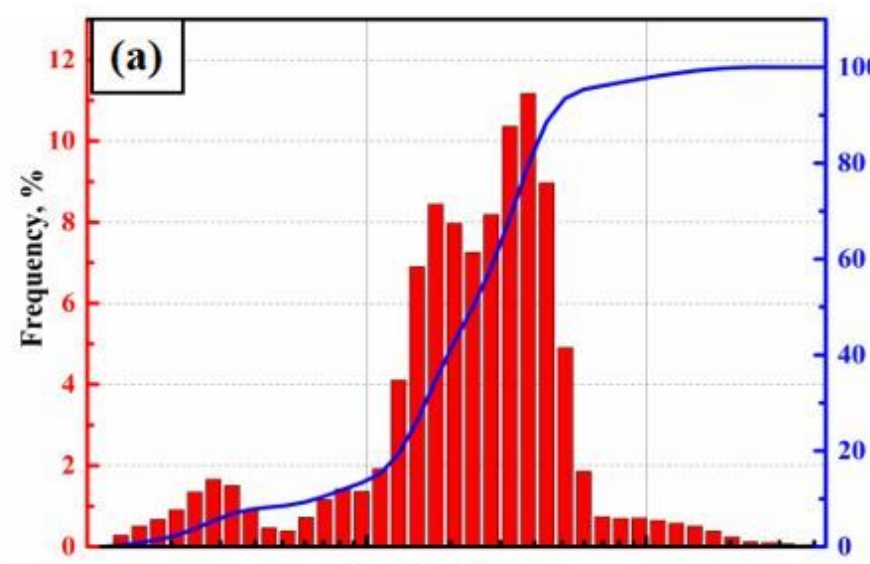

Particle Size, um

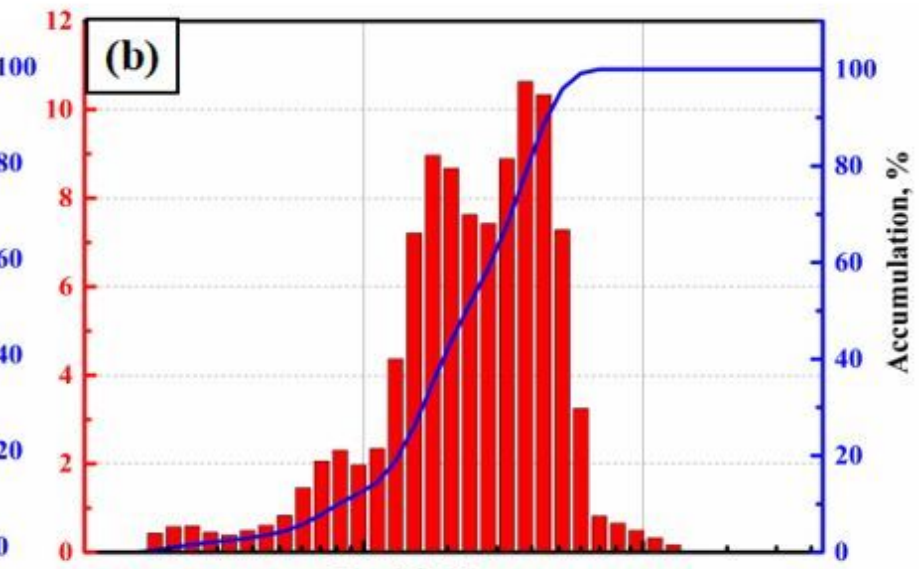

Particle Size,um

Figure 11

particle size distribution of niobium powder before and after oxygen reduction: (a) before pickling, (b) after pickling

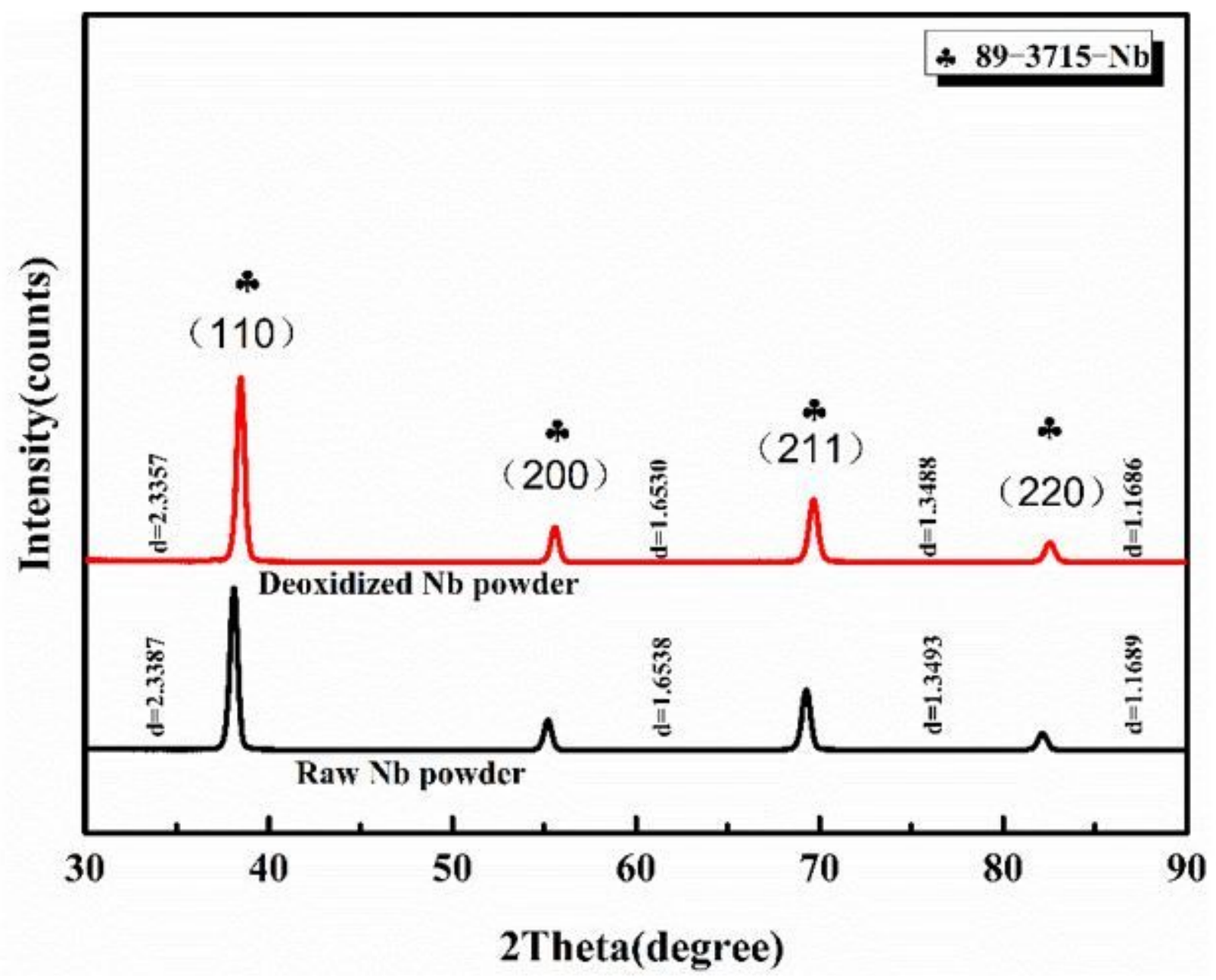

Figure 12 
XRD diffraction pattern of niobium powder before and after deoxidization 